\title{
Long-term visual spectrophotometric behaviour of Be stars $^{\star}$
}

\author{
A. Moujtahid ${ }^{1}$, J. Zorec ${ }^{2}$, A.M. Hubert ${ }^{1}$, A. Garcia ${ }^{2}$, and G. Burki ${ }^{3}$ \\ 1 Observatoire de Paris-Meudon, DASGAL, F-92195 Meudon Principal Cedex, France \\ 2 Institut d'Astrophysique de Paris, CNRS, $98^{\text {bis }}$ Bd. Arago, F-75014 Paris, France \\ e-mail: zorec@iap.fr \\ 3 Observatoire de Genève, CH-1290 Sauverny, Switzerland
}

Received March 11; accepted July 24, 1997

\begin{abstract}
The long-term spectrophotometric variations of $49 \mathrm{Be}$ stars are studied using the $U$ and $V$ magnitudes of the $U B V$ system, the total Balmer discontinuity $D$ and the visible gradient $\Phi_{\mathrm{rb}} . B C D$ spectrophotometric and photometric data in five different photometric systems, obtained in most cases since 1950 and reduced to the $B C D$ system, were used. The $(U, D),(V, D),\left(\Phi_{\mathrm{rb}}, D\right)$ and $\left(\Phi_{\mathrm{rb}}, V\right)$ correlations obtained differ from star to star and they can be single or double-valued. They differ clearly for Be phases or Be-shell phases. Be stars with small $V \sin i$ showing the "spectrophotometric shell behaviour": $D>D_{*}$, were found. This finding implies either that strongly flattened models of circumstellar envelopes are in doubt for these stars, or that not all Be stars are rapid rotators. Comparison of observed variations with those predicted for model Be stars with spherical circumstellar envelopes of variable densities and dimensions implies that spectrophotometric patterns of Be phases are due to circumstellar envelopes in low opacity regimes, while those of spectrophotometric shell phases are due to circumstellar envelopes in high opacity regimes. In a given star, the envelope regions responsible for the observed variations of $D$ and $\Phi_{\mathrm{rb}}$ in spectrophotometric shell phases seem to be smaller and denser than those producing the observed variations of these parameters in spectrophotometric Be phases. The high positive RV found in strong shell phases might favor the formation of compact circumstellar layers near the star.
\end{abstract}

Key words: stars: variable — stars: Be

Send offprint requests to: J. Zorec

* Figure 6 is only available in electronic form at CDS via anonymous ftp to cdsarc.u-strasbg.fr (130.79.128.5) or via http://cdsweb.u-strasbg.fr/Abstract.html

\section{Introduction}

\subsection{General spectrophotometric characteristics of Be stars}

Be stars have long been known to be photometric variables. These variations can have periodic and/or irregular components of different time scales: (a) periodic or multiperiodic components with small amplitudes of roughly several hundredths of magnitude in the $V$ magnitude, characterized by short time scales $t \sim 0.4$ to 3 days (Balona 1990, 1995) which are commonly interpreted as due to non-radial pulsations and/or to stellar surface spots or corotating features (Baade \& Balona 1994); (b) periodic variations with intermediate time scales, $t \sim$ days to 100 days, with amplitudes $\Delta V$ of several hundredths or tenths of magnitude (Harmanec \& Kříž 1976) which are observed only in binary Be stars; (c) quasicyclic or irregular variations with rather long time scales, months, years and sometimes decades, generally implying magnitude changes $\Delta V$ that range between 0.01 and 0.4 mag (Feinstein 1970, 1975; Feinstein \& Marraco 1979; Alvarez \& Schuster 1981; Kozok 1985; Percy et al. 1988). The latter can, however, be as high as $\Delta V \sim 0.5$ to 1.2 mag (Huffer 1939; Mook et al. 1974; Howarth 1979; Alvarez \& Schuster 1981; Bernacca \& Bianchi 1981; Divan et al. 1982, 1983; Mennickent \& Vogt 1991; Apparao 1991; Roche et al. 1993; Mennickent et al. 1994; Ballereau et al. 1995). Though the irregular fluctuations are, as a rule, characterized by long time scales, these scales have been found in the case of very active Be stars to be as short as a few days (Hubert-Delplace et al. 1982). The quasi-cyclic or irregular photometric variations of Be stars are accompanied by more general spectrophotometric variations which concern a wide wavelength coverage. These variations have also been known for a long time (Chalonge \& Safir 1936; Greaves \& Martin 1938; Barbier \& Chalonge 1941). Since Gerasimovic (1929); Chalonge \& Safir (1936); Barbier \& Chalonge (1939), it is also known that when line emission 
or "shell" features are pronounced, Be stars can present two Balmer discontinuities (hereafter $B D$ ). The first $B D$ looks like that of a normal $\mathrm{B}$ star and it has the same spectral location. The second $B D$ appears at shorter wavelengths, which means that it is formed in a medium with a lower pressure than in the photosphere of the central star. It can be in emission (during a strong emission line phase) or in absorption (during a strong "shell" or absorption line phase). The value of the second $B D$ in emission not only depends on the spectral type (Peton 1981) but is correlated with Balmer emission line strength (Schild 1978; Feinstein \& Marraco 1979; Peton 1981; Kaiser 1989; Dachs et al. 1989) and with Balmer decrement as well (Divan et al. 1982). The emission component of the $B D$ is generally not higher than -0.15 dex, but may exceptionally be as high as -0.25 dex (Barbier 1947). When the second $B D$ is in absorption, it can sometimes represent a flux deficiency stronger than $1 \mathrm{mag}$ (Zorec 1986, 1994). The second $B D$ disappears during a faint emission/shell line phase, so that the stellar continuum energy distribution near the $B D$ looks like that of a normal B star. During the spectrophotometric variations of Be stars, the first $B D$ maintains the value and mean spectral position measured during an emissionless phase to closer than 0.02 dex and $\Delta \lambda=1 \AA$ respectively (Divan \& Zorec 1982a,b). The constancy of the first $B D$ to within 0.03 dex was also confirmed by Dachs et al. (1989) and it is currently used for spectral type classification (Divan 1979; Divan \& Zorec 1982c; Divan et al. 1982, 1983). Therefore, it is also useful to determine the effective temperature of the central object (Divan \& Zorec 1982c; Zorec et al. 1983; Zorec 1985, 1986; Kaiser 1989; Dachs et al. 1989; Zorec \& Briot 1991) and the surface gravity (Divan \& Zorec 1982a; Zorec 1985, 1986).

It has been shown that the irregular photometric variations imply a global continuum flux excess or deficiency as compared to stellar underlying photospheric radiation. This can be estimated as $\Delta F_{\mathrm{c}} / F_{\mathrm{c}} \lesssim 10^{-0.4 \Delta V}$, where $\Delta V=V-V_{*}$ ( $V$ magnitude of the star+envelope system; $V_{*}$ magnitude of the star alone) and it is $\Delta V \lesssim 0$ for continuum emission (or flux excess) and $\Delta V \gtrsim 0$ for continuum flux deficiency. The magnitude excess $\Delta V$ is positively correlated with Balmer line emission strength (Zorec \& Briot 1985, 1991; Dachs et al. 1988, 1989; Ballereau et al. 1995) and with reddening of the Paschen continuum (Divan et al. 1978; Divan 1979; Chkhikvadze 1980; Dachs 1982). In turn, this reddening is correlated with Balmer line emission strength (Dachs et al. 1988). The visible flux deficiency is generally small $(\Delta V \lesssim 0.2$ $\mathrm{mag}$ ) and it is not accompanied by marked changes of visible Paschen energy distribution (Divan \& Zorec 1982c; Zorec et al. 1989; Zorec 1994). Quasi-cyclic and/or irregular changes from positive to negative flux excesses are symptomatic of the known Be "phase" variations $\mathrm{Be} \rightleftharpoons$ $\mathrm{B}$-normal $\rightleftharpoons$ Be-shell. The present paper is devoted to the irregular spectrophotometric variations of Be stars.

\subsection{Some previous studies of long-term photometric and/or spectrophotometric variations of Be stars}

The existing observational studies on long-term photometric and/or spectrophotometric variations of Be stars can be classified into two broad groups: (1) studies where authors used observations of their own; (2) studies based on compilations of published observations each made in a given photometric or spectrophotometric system.

(1) - The most relevant studies in the first group are those of Pavlowski et al. (1996) for northern Be stars and Manfroid et al. 1991, 1995; Sterken et al. 1992, 1993 for southern ones. Observations presented by Pavlowski et al. (1996), done in the $U B V$ system, concern the monitoring of 76 Be stars over 18 years since 1972. In this program there are 28 well-observed stars with $9_{-3}^{+8}$ observing seasons and each with $392_{-288}^{+805}$ individual observations, and 48 less observed objects with $3_{-2}^{+2}$ observing seasons each and with $60_{-57}^{+159}$ individual $U B V$ measurements. Manfroid's et al. 1991, 1995 and Sterken's et al. (1992, 1993) 12-year observing program also concerns, among other variable stars, 15 Be stars which were monitored in the uvby Strömgren system since 1982 for about 6 months per year and with a time frequency between 2 and 14 days. From Pavlovski's et al. (1996) study it appears that the number of stars with variations on time scales from months to years is about the same as the number with time scales of days or fractions of days. These variations are, however, 3 times more frequent among stars earlier than B5 than in the later ones. Six stars present orbital components of photometric variations and only 2 show sudden light changes. From the published light curves, which in many cases reveal complicated variations difficult to summarize, we can distinguish those with shell-type characteristics, where two dominant behaviours seem to appear: (i) those of stars where the $(U-B)$ colour index changes much less than the $(B-V)$ index; (ii) those, the most numerous, where the $(U-B)$ colour index changes much more than the $(B-V)$ index. Both types of behaviours are seen in early as well as in late type Be stars. Mennickent et al. (1994) report a detailed study on 13 Be stars based on uvby Manfroid \& Sterken's et al. 1991-1995 observations. These authors note: (a) quasi-periodic oscillations on time scales between 10 and 20 days with amplitudes up to $0.2 \mathrm{mag}$ preceding fading events; (b) in 7 stars, which mostly have shell characteristics, they observe that: $\left(b_{1}\right)$ small or rather no variations of the $b-y$ index accompany the variation of magnitude $u$; $\left(\mathrm{b}_{2}\right)$ there is a correlation between index $c_{1}$ and magnitude $u$; (c) in all 13 studied Be stars and for all uvby magnitudes, there is, within the uncertainties of coefficients defining the regression lines, a mean relation between the long-term variation amplitudes and the standard deviation of short-term variability. Concerning the $\left(c_{1}, u\right)$ relation, it is worth noting that in shell Be stars not only the variation of $u$ is noticeably higher than in $v$ but also $(b-v) \sim 0$, so that the 
correlation between $c_{1}$ and $u$ seems natural. However, the well-defined trend between the slope of the $\left(c_{1}, u\right)$ relation and $V \sin i$, which identifies possible differences in the relative behaviours of Balmer to Paschen continua, could still deserve further attention.

The first group also comprises studies of long-term spectrophotometric variations of Be stars made using the $B C D$ system (for definition of the $B C D$ (BarbierChalonge-Divan) system, see Chalonge \& Divan 1952). The great advantage of this system is that it reflects the variation of continuum energy distribution only, because it is not affected by spurious "reddening/blueing" effects introduced by variable spectral lines entering the wide wavelength intervals of some photometric filter pass-bands. The $B C D$ data were obtained at irregular time intervals over more than 40 years. They concern a small number of Be stars, of which most are of early spectral types. These data led Divan (1979); Divan et al. (1982); Zorec (1986) to conclude that in the studied objects the continuum emission phases were characterized by well-defined relations between the visible gradients of energy distributions, total Balmer discontinuity and visual magnitude. These relations seem to be different for each star and unchanged even after the star had a phase of apparent loss of emission characteristics. These authors also found that, in general, reddening of the visible gradient is positively correlated with stellar brightening. Inversely, "shell" phases are characterized by very small changes, if any, in the visible energy distribution.

(2) - In the second group are papers concerning either a small number of objects at typical "Be" phases, or a large number of objects at any variation phase. In the first subgroup, works like Dachs (1982); Dachs \& Hanuschik (1984) and Dachs et al. (1988) show that long-term photometric variations can be characterized by slopes $\Delta V / \Delta(B-V)$ ranging between about -0.1 and -0.3 for individual stars and that reddening of the $(B-V)$ index is related to stellar brightening. In the second subgroup, it is worth mentioning Nordh \& Olofsson (1977); Hirata (1982) and Hirata \& Hubert-Delplace (1981). From $U B V$ data of 50 to $70 \mathrm{Be}$ stars spread over more than 10 years, Hirata et al. concluded: (i) that early type Be stars get redder when they brighten, and on the contrary late type Be stars are bluer when they brighten; (ii) the slope $\Delta V / \Delta(B-V)$ is steeper when $V \sin i$ is higher, and that this slope has the same sign as the colour excess ratio $\Delta(U-B) / \Delta(B-V)$.

Finally, there are some attempts of a theoretical nature to explain the long-term photometric variations of Be stars. Using a cylindrical model for the CE, Hirata (1984) concluded that changes of $V$ and $(B-V)$ are due to variations of the extent of the circumstellar envelope (hereafter $\mathrm{CE}$ ) and that $\Delta V / \Delta(B-V)$ is a function of spectral type and aspect angle. The irregular variations of $V$ magnitude were also discussed by Zorec \& Briot (1991). They concluded that when $\Delta V<0$, the irregular variations may correspond to two different opacity regimes of the circumstellar envelope: (a) a low opacity regime, where variations of $V$ are mostly due to small changes of opacity, and (b) a high opacity regime, where variations of $V$ are mainly due to changes of the extent of the CE. Inversely, for $\Delta V>0$ there is only a high opacity regime. In a recent work, Hirata (1995) studied the long-term variations of some Be stars at shell phases and concluded that their variations are produced by extended photospheres.

As continuum and line variations correlate only when strong line emissions or line absorptions are present during "spectroscopic" Be and Be-shell phases respectively, to avoid misunderstandings relative to spectroscopic or spectrophotometric variations, whenever necessary we explicitly refer in this paper to "spectrophotometric Be phase" (hereafter $\mathrm{SPh}-\mathrm{Be}$ ) when the second $B D$ is in emission and to "spectrophotometric shell phase" (hereafter SPh-shell) when the second $B D$ is in absorption.

\subsection{Aim of the present work}

Results presented in the preceding section are consistent and complementary to each other. The relations obtained between photometric colour indices and/or spectrophotometric parameters show that they should somehow depend on the physical structure of the $\mathrm{CE}$ and on its changes. In particular, early $B C D$ results suggested that these relations are permanent for each star and differ from one object to another, as would be the case if there were permanent characteristics related to the structure of the $\mathrm{CE}$ of Be stars which subsist even after phase changes or apparent losses of emission. However, in addition to opacity and geometrical factors cited in the preceding section, still other phenomena could be responsible for photometric/spectrophotometric changes of Be stars. In fact, as the contributing regions in the $\mathrm{CE}$ to the $\Delta V$ variations are expected to be located rather near the underlying star (Zorec \& Briot 1991; Zorec \& Garcia 1991; Cruzado et al. 1994) ionization/excitation balance changes in the deepest layers of the CE (Magnan 1979), as well as radiation transfer effects related to non-thermal phenomena (Gebbie \& Thomas 1970, 1971; Thomas 1983) should also be relevant. Due to all these effects and because some of the above observational results were obtained from a small number, mostly early type Be stars, it would still be necessary: (a) to analyse a larger sample of Be stars of all possible subspectral types, each having been observed as frequently as possible and over a long time base; (b) to study the actual character (constancy, uniqueness, etc.) of relations among spectrophotometric parameters for each star, especially, if feasible, for those having undergone phase changes. This study aims at giving as far as possible comprehensive observational bases on long-term spectrophotometric variations of Be stars, derived from compiled photometric and spectrophotometric data obtained in various systems, to ensure a large time base coverage and for a quite a large number of stars. 


\section{Method}

\subsection{General remarks}

To compare more or less heterogeneous photometric and spectrophotometric data, obtained at different epochs by different observers and systems, we reduced them all to a common set of spectrophotometric quantities. We transformed them to the spectrophotometric parameters derived in the $B C D$ system. These are: (a) gradient $\Phi_{\mathrm{rb}}$ of the continuum energy distribution in the spectral domain $\lambda \lambda 0.40-0.63 \mu \mathrm{m}$; (b) total $B D$ of Be stars, D, measured at $\lambda 0.37 \mu \mathrm{m}$, and (c) $V$ magnitude as defined in the standard $U B V$ system. As known, the total $B D$ of Be stars has two components: $D=D_{*}+d$, where $D_{*} \geq 0$ accounts for the invariable stellar $B D$, and $d$ is the variable component due to the CE. It is $d<0$ for Balmer continuum emission excess, and $d>0$ for Balmer continuum deficiency. In this paper we study the total $B D$ only.

\subsection{The $\left(\Phi_{\mathrm{rb}}, D, V\right)$ parameters and the structure of the $C E$}

Reduction of all data obtained from quite different photometric systems to the $B C D$ parametric triptych $\left(\Phi_{\mathrm{rb}}\right.$, $D, V)$, allows us: (a) to have an uniform set of parameters with a clear physical meaning and easy to interpret theoretically; (b) to test previous conclusions obtained with the $B C D$ data on long-term spectrophotometric behaviour of Be stars. Interpretation of the $\left(\Phi_{\mathrm{rb}}, D, V\right)$ parameters in terms of CE opacity, extent and temperature will be sketched in Sect. 4 .

\subsection{Photometric systems of compiled data}

Data of Be stars used in this work were obtained in (or reduced in the original publications to) the following photometric systems: (a) the $U B V$ standard system (Johnson \& Morgan 1951, 1953); (b) the UBVRI system (Johnson 1965; Cousins 1976, 1978); (c) the sevencolour $(U, B, V, B 1, B 2, V 1, G)$ Geneva photometric system (Golay 1963; Rufener \& Maeder 1971; Rufener \& Nicolet 1988; Rufener 1979); (d) the uvby Strömgren system (Strömgren 1966; Grønbech et al. 1976; Grønbech \& Olsen 1977); (e) 13-colour photometry (Johnson \& Mitchell 1975); (f) photographic spectrophotometric data $(B C D)$.

\subsection{The stellar sample}

From all known Be stars (Jaschek \& Egret 1982) we have chosen the brightest ones because they were liable to yield more frequent and detailed observations. Among them we preferred those known for their strong spectroscopic variations (Hubert-Delplace \& Hubert 1979) or because they had rather strong photometric changes (Feinstein 1975; Feinstein \& Marraco 1979; Alvarez \& Schuster 1981). We discarded those objects in binaries where variations of Be characteristics are strongly correlated with the orbital phase, such as HR 2142. The studied Be stars are presented in Table 1. The spectral classification is preferentially from the $B C D$ system and in such cases it is indicated with an upper index " $a$ ". Otherwise, it is taken from the Hipparcos Input Catalogue (Turon et al. 1992); Buscombe (1977, 1980, 1981, 1988); Hubert-Delplace \& Hubert (1979); Slettebak (1982), the Bright Stars Catalogue (Hoffleit \& Jaschek 1982) and the Supplement to the Bright Stars Catalogue (Hoffleit et al 1983). For each Be star are given: the stellar $B D, D_{*}$ (an upper index " $a$ " indicates $B C D$ values of $D_{*}$, otherwise $D_{*}$ is a mean value for the adopted MK spectral classification); the mean values of $V, D$ and $\Phi_{\mathrm{rb}}$ calculated from all collected data and the respective standard deviations $\left(\sigma_{V}, \sigma_{D}, \sigma_{\Phi}\right)$; the JD-2400000 of the first and the last observation; the number of individual triads $\left(V, D, \Phi_{\mathrm{rb}}\right)$; the references of collected data. The standard deviation of variations in the $(B-V)$ and $(U-B)$ colour indices can straightforwardly be derived using the transformations: $\sigma_{(\mathrm{B}-\mathrm{V})}=0.56 \sigma_{\Phi}$ and $\sigma_{(\mathrm{U}-\mathrm{B})}^{2}=3.89 \sigma_{\mathrm{D}}^{2}+0.54 \sigma_{\Phi}^{2}$.

\subsection{Reduction to spectrophotometric parameters}

\subsubsection{Comparison stars}

We took as comparison stars a set of 82 well-observed $\mathrm{O}$, $\mathrm{B}$ and $\mathrm{A} 0$ type stars in the $B C D$ system, all presented in Zorec \& Briot (1991), whose $\Phi_{\mathrm{rb}}$ and $D$ parameters are determined to closer than $\delta\left(\Phi_{\mathrm{rb}}\right)=0.05 \mu \mathrm{m}$ and $\delta(D)=0.01$ dex respectively. We considered stars of luminosity classes from dwarfs to bright giants and with different rotational velocities to get a first and direct insight on the possible scatter that differences in intensities of spectral lines can introduce in the transformations (photometric indices) $\rightarrow$ (spectrophotometric parameters). A large number of these stars $(75 \%)$ are used either as MK, photometric or spectrophotometric standard/comparison stars (Divan 1966; Golay 1973; Rufener 1979; Hauck 1985; Tüg 1980; Thé et al. 1986; Perry et al. 1987; Zorec et al. 1983; Harmanec et al. 1994). Table 2 gives the adopted comparison stars, with the $\Phi_{\mathrm{rb}}$ and $D$ parameters and the corresponding MK spectral type as derived using the $B C D$ system.

\subsubsection{Transformation of photometric data into the $\Phi_{\mathrm{rb}}$} and $D$ spectrophotometric parameters

\section{i) The $U B V$ system.}

The best correlation between the $U B V$ colour indices and the $B D$ given in the $B C D$ system was found using Johnson's (1958) $Q$ index, which leads, as in the $B C D$ system, to $B D$ free from interstellar medium (ISM) absorption. We used the definition of the $Q$ index given by Gutierrez-Moreno (1975):

$Q_{U B V}=(U-B)-0.67(B-V)$ mag. 
Table 1. The studied Be stars

\begin{tabular}{|c|c|c|c|c|c|c|c|c|c|}
\hline $\mathrm{HD}$ & $\begin{array}{l}\text { HR } \\
\text { Name }\end{array}$ & Sp.Type & $\begin{array}{l}D_{*} \\
\operatorname{dex}\end{array}$ & $\begin{array}{l}\bar{V} \\
\sigma_{V}\end{array}$ & $\begin{array}{r}\bar{D} \\
\sigma_{D}\end{array}$ & $\begin{array}{r}\overline{\Phi_{\mathrm{rb}}} \\
\sigma_{\Phi}\end{array}$ & $\mathrm{JD}_{\mathrm{f}}-\mathrm{JD}_{\mathrm{l}}$ & $N$ & References \\
\hline 4180 & $\begin{array}{c}193 \\
o \text { Cas }\end{array}$ & B5-6IIIe ${ }^{a}$ & $0.270^{a}$ & $\begin{array}{l}4.55 \\
0.06\end{array}$ & $\begin{array}{l}0.261 \\
0.006\end{array}$ & $\begin{array}{l}1.00 \\
0.02\end{array}$ & $35400-42021$ & 17 & $\begin{array}{l}10,15,32,34,38,80,91,110 \\
114,115,142,198\end{array}$ \\
\hline 5394 & $\begin{array}{c}264 \\
\gamma \text { Cas }\end{array}$ & O9Ve ${ }^{a}$ & $0.04:{ }^{a}$ & $\begin{array}{l}2.41 \\
0.22\end{array}$ & $\begin{array}{r}-0.038 \\
0.054\end{array}$ & $\begin{array}{l}0.97 \\
0.13\end{array}$ & $28070-47528$ & 389 & $\begin{array}{l}7,12,13,14,15,32,38,67,80 \\
101,104,109,110,111,112 \\
114,115,142,155,198\end{array}$ \\
\hline 23862 & $\begin{array}{c}1180 \\
28 \mathrm{Tau}\end{array}$ & $\mathrm{B} 8 \mathrm{IV}-\mathrm{Ve}^{a}$ & $0.370^{a}$ & $\begin{array}{l}5.17 \\
0.09\end{array}$ & $\begin{array}{l}0.399 \\
0.079\end{array}$ & $\begin{array}{l}1.01 \\
0.06\end{array}$ & $33976-48341$ & 341 & $\begin{array}{l}2,11,12,13,14,19,33,35,42, \\
53,56,65,80,85,99,100,110, \\
114,115,116,158,172,178,196 \\
197,198\end{array}$ \\
\hline 24534 & $\begin{array}{r}1209 \\
\mathrm{X} \text { Per }\end{array}$ & O9IVe ${ }^{a}$ & $0.050^{a}$ & $\begin{array}{l}6.55 \\
0.21\end{array}$ & $\begin{array}{l}0.000 \\
0.045\end{array}$ & $\begin{array}{l}1.44 \\
0.12\end{array}$ & $34271-49029$ & 331 & $\begin{array}{l}2,13,17,18,38,43,66,67,69 \\
87,94,95,105,106,118,127 \\
141,142,147,151,164,198\end{array}$ \\
\hline 25940 & $\begin{array}{l}1273 \\
48 \text { Per }\end{array}$ & B4IVe ${ }^{a}$ & $0.230^{a}$ & $\begin{array}{l}4.04 \\
0.02\end{array}$ & $\begin{array}{l}0.225 \\
0.013\end{array}$ & $\begin{array}{l}1.06 \\
0.02\end{array}$ & $35400-40560$ & 18 & $\begin{array}{l}32,38,80,81,82,87,110,114, \\
115,142,144,198\end{array}$ \\
\hline 28497 & $\begin{array}{c}1423 \\
\text { DU Eri }\end{array}$ & $\mathrm{B} 1.5 \mathrm{Ve}^{a}$ & $0.124^{a}$ & $\begin{array}{l}5.51 \\
0.04\end{array}$ & $\begin{array}{l}0.066 \\
0.016\end{array}$ & $\begin{array}{l}0.81 \\
0.03\end{array}$ & $35400-48202$ & 92 & $\begin{array}{l}6,26,37,58,64,68,73,110, \\
142,157,198\end{array}$ \\
\hline 32343 & $\begin{array}{c}1622 \\
11 \text { Cam }\end{array}$ & $\mathrm{B} 4 \mathrm{Ve}^{a}$ & $0.223^{a}$ & $\begin{array}{l}5.13 \\
0.09\end{array}$ & $\begin{array}{l}0.152 \\
0.023\end{array}$ & $\begin{array}{l}0.97 \\
0.02\end{array}$ & $32809-45697$ & 25 & $\begin{array}{l}38,51,110,128,142,155,196 \\
198\end{array}$ \\
\hline 33328 & $\begin{array}{l}1679 \\
\lambda \text { Eri }\end{array}$ & B2III-IVe ${ }^{a}$ & $0.145^{a}$ & $\begin{array}{l}4.26 \\
0.02\end{array}$ & $\begin{array}{l}0.113 \\
0.006\end{array}$ & $\begin{array}{l}0.79 \\
0.02\end{array}$ & $37666-49278$ & 404 & $\begin{array}{l}3,26,30,38,68,78,110,114, \\
115,139,140,159,181,182,198\end{array}$ \\
\hline 35439 & $\begin{array}{c}1789 \\
25 \text { Ori }\end{array}$ & B1.5III-IVe ${ }^{a}$ & $0.121^{a}$ & $\begin{array}{l}4.80 \\
0.10\end{array}$ & $\begin{array}{l}0.065 \\
0.026\end{array}$ & $\begin{array}{l}0.86 \\
0.07\end{array}$ & $33966-48313$ & 117 & $\begin{array}{l}2,8,22,30,38,73,80,110,114, \\
115,126,142,143,174,191,198\end{array}$ \\
\hline 37202 & $\begin{array}{l}1910 \\
\zeta \text { Tau }\end{array}$ & B2IIIe ${ }^{a}$-shell & $0.142^{a}$ & $\begin{array}{l}2.97 \\
0.05\end{array}$ & $\begin{array}{l}0.178 \\
0.026\end{array}$ & $\begin{array}{l}0.80 \\
0.04\end{array}$ & $35400-48974$ & 78 & $\begin{array}{l}32,38,59,76,80,85,110,114 \\
115,142,198\end{array}$ \\
\hline 37967 & $\begin{array}{c}1961 \\
\text { V731 Tau }\end{array}$ & $\mathrm{B} 4 \mathrm{Ve}^{a}$ & $0.220^{a}$ & $\begin{array}{l}6.23 \\
0.05\end{array}$ & $\begin{array}{l}0.191 \\
0.012\end{array}$ & $\begin{array}{l}1.00 \\
0.03\end{array}$ & $35400-48600$ & 26 & $32,38,85,110,142,193,198$ \\
\hline 48914 & & B5Ib-II & 0.200 & $\begin{array}{l}7.27 \\
0.07\end{array}$ & $\begin{array}{l}0.300 \\
0.035\end{array}$ & $\begin{array}{l}1.12 \\
0.03\end{array}$ & $41329-47934$ & 640 & $55,184,187,198$ \\
\hline 48917 & $\begin{array}{c}2492 \\
10 \mathrm{CMa}\end{array}$ & B2III-IVe ${ }^{a}$ & $0.147^{a}$ & $\begin{array}{l}5.21 \\
0.06\end{array}$ & $\begin{array}{l}0.069 \\
0.015\end{array}$ & $\begin{array}{l}0.93 \\
0.04\end{array}$ & $38441-49407$ & 406 & $\begin{array}{l}22,37,45,57,59,60,62,73,139, \\
140,179,181,182,185,198\end{array}$ \\
\hline 50123 & 2545 & B5IIIe $^{a}$ & $0.220^{a}$ & $\begin{array}{l}5.75 \\
0.03\end{array}$ & $\begin{array}{l}0.158 \\
0.008\end{array}$ & $\begin{array}{l}1.36 \\
0.03\end{array}$ & $40967-49401$ & 186 & $\begin{array}{l}73,92,125,139,140,179,181 \\
182,198\end{array}$ \\
\hline 56014 & $\begin{array}{c}2745 \\
27 \mathrm{CMa}\end{array}$ & B3IIIe $^{a}$ & $0.190^{a}$ & $\begin{array}{l}4.59 \\
0.08\end{array}$ & $\begin{array}{l}0.146 \\
0.030\end{array}$ & $\begin{array}{l}0.81 \\
0.03\end{array}$ & $38104-47232$ & 182 & $\begin{array}{l}6,22,58,60,62,73,114,139 \\
140,181,182,198\end{array}$ \\
\hline 56139 & $\begin{array}{c}2749 \\
\omega \mathrm{CMa}\end{array}$ & $\mathrm{B} 2 \mathrm{IVe}^{a}$ & $0.165^{a}$ & $\begin{array}{l}3.77 \\
0.17\end{array}$ & $\begin{array}{l}0.164 \\
0.030\end{array}$ & $\begin{array}{l}0.92 \\
0.08\end{array}$ & $38384-48349$ & 182 & $\begin{array}{l}6,22,26,45,59,60,62,78,108 \\
114,139,140,179,181,198\end{array}$ \\
\hline 58050 & 2817 & $\mathrm{~B} 2 \mathrm{Ve}^{a}$ & $0.160^{a}$ & $\begin{array}{l}6.56 \\
0.09\end{array}$ & $\begin{array}{l}0.145 \\
0.010\end{array}$ & $\begin{array}{l}0.76 \\
0.02\end{array}$ & $35400-48344$ & 41 & $38,110,142,163,170,198$ \\
\hline 58978 & $\begin{array}{l}2855 \\
\text { FY CMa }\end{array}$ & $\mathrm{B} 0 \mathrm{Ve}^{a}$ & $0.07:^{a}$ & $\begin{array}{l}5.62 \\
0.04\end{array}$ & $\begin{array}{l}0.031 \\
0.006\end{array}$ & $\begin{array}{l}0.95 \\
0.02\end{array}$ & $38135-49399$ & 275 & $\begin{array}{l}1,22,24,37,57,58,59,62,64 \\
73,74,91,131,139,140,179 \\
181,182,198\end{array}$ \\
\hline 60848 & BN Gem & O8Vpe ${ }^{a}$ & $0.050^{a}$ & $\begin{array}{l}6.85 \\
0.07\end{array}$ & $\begin{array}{l}0.009 \\
0.027\end{array}$ & $\begin{array}{l}0.85 \\
0.06\end{array}$ & $35571-48347$ & 14 & $48,74,135,198$ \\
\hline 60855 & 2921 & B3IVe & 0.190 & $\begin{array}{l}5.69 \\
0.03\end{array}$ & $\begin{array}{l}0.171 \\
0.011\end{array}$ & $\begin{array}{l}0.91 \\
0.03\end{array}$ & $41484-45746$ & 19 & $\begin{array}{l}26,31,37,59,73,97,134,136 \\
142,169,170,175,198\end{array}$ \\
\hline 63462 & $\begin{array}{c}3034 \\
\text { o Pup }\end{array}$ & $\mathrm{B} 0 \mathrm{Ve}^{a}$ & $0.04:{ }^{a}$ & $\begin{array}{l}4.49 \\
0.02\end{array}$ & $\begin{array}{r}-0.000 \\
0.007\end{array}$ & $\begin{array}{l}1.25 \\
0.02\end{array}$ & $38076-49439$ & 180 & $\begin{array}{l}26,30,36,58,59,73,98,114 \\
140,143,179,181,182,198\end{array}$ \\
\hline 65875 & 3135 & $\mathrm{~B}^{2} \mathrm{IVe}^{a}$ & $0.140^{a}$ & $\begin{array}{l}6.51 \\
0.05\end{array}$ & $\begin{array}{l}0.127 \\
0.023\end{array}$ & $\begin{array}{l}0.94 \\
0.05\end{array}$ & $35400-48178$ & 23 & $\begin{array}{l}38,58,59,73,110,132,142, \\
170,187,189,198\end{array}$ \\
\hline 68980 & $\begin{array}{l}3237 \\
\text { MX Pup }\end{array}$ & $\mathrm{B} 0.5 \mathrm{IV}-\mathrm{Ve}^{a}$ & $0.090^{a}$ & $\begin{array}{l}4.73 \\
0.09\end{array}$ & $\begin{array}{l}0.036 \\
0.016\end{array}$ & $\begin{array}{l}0.94 \\
0.04\end{array}$ & $38076-49399$ & 155 & $\begin{array}{l}26,30,58,59,60,61,62,64,78 \\
139,140,179,181,182,192,198\end{array}$ \\
\hline 83953 & 3858 & $\mathrm{~B} 5 \mathrm{IV}-\mathrm{Ve}^{a}$ & $0.260^{a}$ & $\begin{array}{l}4.76 \\
0.02\end{array}$ & $\begin{array}{l}0.238 \\
0.011\end{array}$ & $\begin{array}{l}0.89 \\
0.02\end{array}$ & $35400-48972$ & 66 & $\begin{array}{l}8,26,30,36,58,59,64,73,78 \\
114,129,140,142,150,179 \\
181,182,198\end{array}$ \\
\hline 89890 & 4074 & B4III-IVe & 0.235 & $\begin{array}{l}4.49 \\
0.01\end{array}$ & $\begin{array}{l}0.252 \\
0.007\end{array}$ & $\begin{array}{l}0.91 \\
0.02\end{array}$ & $37666-49400$ & 153 & $\begin{array}{l}26,30,36,44,64,73,139,140 \\
179,181,182,198\end{array}$ \\
\hline
\end{tabular}


Table 1. continued

\begin{tabular}{|c|c|c|c|c|c|c|c|c|c|}
\hline HD & $\begin{array}{c}\text { HR } \\
\text { Name }\end{array}$ & Sp.Type & $\begin{array}{l}D_{*} \\
\operatorname{dex}\end{array}$ & $\begin{array}{l}\overline{\bar{V}} \\
\sigma_{V}\end{array}$ & $\begin{array}{r}\bar{D} \\
\sigma_{D}\end{array}$ & $\begin{array}{r}\overline{\Phi_{\mathrm{rb}}} \\
\sigma_{\Phi}\end{array}$ & $\mathrm{JD}_{\mathrm{f}}-\mathrm{JD}_{\mathrm{l}}$ & $N$ & References \\
\hline 91120 & 4123 & B9IVe ${ }^{a}$ & $0.430^{a}$ & $\begin{array}{l}5.60 \\
0.01\end{array}$ & $\begin{array}{l}0.450 \\
0.008\end{array}$ & $\begin{array}{l}1.04 \\
0.02\end{array}$ & $37666-48219$ & 22 & $23,33,73,110,170,183,198$ \\
\hline 91465 & $\begin{array}{c}4140 \\
\text { PP car }\end{array}$ & B3IIIe $^{a}$ & $0.187^{a}$ & $\begin{array}{l}3.31 \\
0.08\end{array}$ & $\begin{array}{l}0.166 \\
0.013\end{array}$ & $\begin{array}{l}0.90 \\
0.04\end{array}$ & $38807-48258$ & 23 & $23,27,36,58,179,181,198$ \\
\hline 109387 & $\begin{array}{c}4787 \\
\kappa \text { Dra }\end{array}$ & $\mathrm{B} 5-6 \mathrm{IVe}^{a}$ & $0.292^{a}$ & $\begin{array}{l}3.87 \\
0.05\end{array}$ & $\begin{array}{l}0.243 \\
0.020\end{array}$ & $\begin{array}{l}0.88 \\
0.05\end{array}$ & $35400-47110$ & 201 & $\begin{array}{l}2,10,19,20,38,42,75,79,80 \\
110,114,115,117,142,154 \\
170,198\end{array}$ \\
\hline 120991 & $\begin{array}{c}5223 \\
\text { V767 Cen }\end{array}$ & B2IIIe & 0.140 & $\begin{array}{l}6.07 \\
0.14\end{array}$ & $\begin{array}{l}0.063 \\
0.042\end{array}$ & $\begin{array}{l}0.98 \\
0.08\end{array}$ & $38472-48851$ & 229 & $\begin{array}{l}23,37,58,62,63,64,72,73, \\
119,121,124,177,179,194,198\end{array}$ \\
\hline 131492 & $\begin{array}{l}5551 \\
\theta \text { Cir }\end{array}$ & $\mathrm{B} 3 \mathrm{Ve}^{a}$ & $0.187^{a}$ & $\begin{array}{l}5.29 \\
0.17\end{array}$ & $\begin{array}{l}0.165 \\
0.069\end{array}$ & $\begin{array}{l}1.04 \\
0.06\end{array}$ & $38031-48117$ & 52 & $\begin{array}{l}58,62,64,73,77,122,179,185 \\
198\end{array}$ \\
\hline 142983 & $\begin{array}{c}5941 \\
48 \mathrm{Lib}\end{array}$ & $\begin{array}{l}\text { B3-4III-IVe }{ }^{a} \\
\text {-shell }\end{array}$ & $0.212^{a}$ & $\begin{array}{l}4.88 \\
0.07\end{array}$ & $\begin{array}{l}0.334 \\
0.063\end{array}$ & $\begin{array}{l}0.97 \\
0.02\end{array}$ & $38031-49593$ & 595 & $\begin{array}{l}2,6,26,36,58,60,62,64,73 \\
77,110,114,115,139,140,145 \\
170,177,181,182,196,198\end{array}$ \\
\hline 148184 & $\begin{array}{c}6118 \\
\chi \mathrm{Oph}\end{array}$ & $\mathrm{B} 0.5 \mathrm{Ve}^{a}$ & $0.09:^{a}$ & $\begin{array}{l}4.30 \\
0.07\end{array}$ & $\begin{array}{r}-0.011 \\
0.020\end{array}$ & $\begin{array}{l}1.67 \\
0.06\end{array}$ & $35400-48123$ & 175 & $\begin{array}{l}2,6,26,58,62,64,77,83,92, \\
110,114,115,142,170,196,198\end{array}$ \\
\hline 162428 & & B7IV-Ve & 0.345 & $\begin{array}{l}7.12 \\
0.02\end{array}$ & $\begin{array}{l}0.298 \\
0.052\end{array}$ & $\begin{array}{l}0.99 \\
0.03\end{array}$ & $43304-47744$ & 9 & $2,122,169,198$ \\
\hline 162732 & $\begin{array}{c}6664 \\
88 \mathrm{Her}\end{array}$ & B6IVe ${ }^{a}$-shell & $0.300^{a}$ & $\begin{array}{l}6.80 \\
0.07\end{array}$ & $\begin{array}{l}0.341 \\
0.029\end{array}$ & $\begin{array}{l}0.89 \\
0.04\end{array}$ & $37666-49234$ & 197 & $\begin{array}{l}2,5,9,19,33,39,47,49,84,85 \\
89,91,96,110,149,151,160 \\
170,198\end{array}$ \\
\hline 168797 & 6873 & B4IVe & 0.220 & $\begin{array}{l}6.13 \\
0.03\end{array}$ & $\begin{array}{l}0.191 \\
0.011\end{array}$ & $\begin{array}{l}1.06 \\
0.02\end{array}$ & $35400-48328$ & 43 & $\begin{array}{l}4,28,38,54,110,122,142,160 \\
166,198\end{array}$ \\
\hline 171780 & 6984 & B5Vne & 0.264 & $\begin{array}{l}6.10 \\
0.03\end{array}$ & $\begin{array}{l}0.248 \\
0.013\end{array}$ & $\begin{array}{l}0.90 \\
0.02\end{array}$ & $38913-48375$ & 11 & $38,110,142,169,170,178,198$ \\
\hline 173219 & & B0Ve & $0.07:$ & $\begin{array}{l}7.85 \\
0.02\end{array}$ & $\begin{array}{l}0.004 \\
0.005\end{array}$ & $\begin{array}{l}1.49 \\
0.02\end{array}$ & $34271-49594$ & 343 & $\begin{array}{l}88,94,122,139,140,142,181 \\
182,198\end{array}$ \\
\hline 178175 & $\begin{array}{c}7249 \\
\text { V4024 Sgr }\end{array}$ & $\mathrm{B} 2 \mathrm{Ve}$ & 0.162 & $\begin{array}{l}5.54 \\
0.05\end{array}$ & $\begin{array}{l}0.175 \\
0.015\end{array}$ & $\begin{array}{l}1.00 \\
0.02\end{array}$ & $35400-48341$ & 36 & $\begin{array}{l}24,37,58,59,60,62,64,73 \\
110,122,142,198\end{array}$ \\
\hline 183656 & $\begin{array}{l}7415 \\
\text { V923 Aql }\end{array}$ & B6Ve-shell & 0.315 & $\begin{array}{l}6.07 \\
0.03\end{array}$ & $\begin{array}{l}0.378 \\
0.031\end{array}$ & $\begin{array}{l}1.12 \\
0.02\end{array}$ & $39902-49591$ & 162 & $\begin{array}{l}19,28,73,91,139,140,181, \\
182,198\end{array}$ \\
\hline 184279 & V1294 Aql & $\mathrm{B} 0 \mathrm{Ve}$ & $0.06:$ & $\begin{array}{l}7.01 \\
0.11\end{array}$ & $\begin{array}{l}0.092 \\
0.031\end{array}$ & $\begin{array}{l}1.23 \\
0.04\end{array}$ & $34013-49591$ & 340 & $\begin{array}{l}2,29,30,40,91,110,113,122 \\
138,140,142,148,161,162 \\
170,181,181,186,188,198\end{array}$ \\
\hline 187811 & $\begin{array}{c}7565 \\
12 \mathrm{Vul}\end{array}$ & $\mathrm{B} 2.5 \mathrm{Ve}$ & 0.170 & $\begin{array}{l}4.89 \\
0.04\end{array}$ & $\begin{array}{l}0.208 \\
0.012\end{array}$ & $\begin{array}{l}0.82 \\
0.02\end{array}$ & $35400-46664$ & 79 & $\begin{array}{l}38,54,55,110,114,115,130 \\
142,146,160,198\end{array}$ \\
\hline 191610 & $\begin{array}{c}7708 \\
28 \mathrm{Cyg}\end{array}$ & B3IVe & 0.190 & $\begin{array}{l}4.93 \\
0.03\end{array}$ & $\begin{array}{l}0.144 \\
0.012\end{array}$ & $\begin{array}{l}0.85 \\
0.03\end{array}$ & $35400-48441$ & 192 & $\begin{array}{l}1,19,38,54,71,114,115,130 \\
142,146,156,160,198\end{array}$ \\
\hline 195325 & $\begin{array}{l}7836 \\
1 \text { Del }\end{array}$ & B9e-Shell & $0.44:$ & $\begin{array}{l}6.03 \\
0.04\end{array}$ & $\begin{array}{l}0.485 \\
0.038\end{array}$ & $\begin{array}{l}1.08 \\
0.02\end{array}$ & $39902-48372$ & 11 & $19,91,110,153,171,198$ \\
\hline 200120 & $\begin{array}{c}8047 \\
59 \mathrm{Cyg}\end{array}$ & $\mathrm{B} 1.5 \mathrm{Ve}^{a}$ & $0.116^{a}$ & $\begin{array}{l}4.80 \\
0.08\end{array}$ & $\begin{array}{l}0.052 \\
0.016\end{array}$ & $\begin{array}{l}0.95 \\
0.06\end{array}$ & $32833-49719$ & 660 & $\begin{array}{l}2,10,15,38,46,50,52,107 \\
110,114,115,130,133,134 \\
142,155,160,167,196,198\end{array}$ \\
\hline 205637 & $\begin{array}{c}8260 \\
\epsilon \text { Cap }\end{array}$ & $\begin{array}{l}\text { B3II-IIIe }{ }^{a} \\
\text {-shell }\end{array}$ & $0.155^{a}$ & $\begin{array}{l}4.54 \\
0.06\end{array}$ & $\begin{array}{l}0.203 \\
0.026\end{array}$ & $\begin{array}{l}0.79 \\
0.02\end{array}$ & $35400-49593$ & 449 & $\begin{array}{l}22,26,28,30,58,59,60,64,73 \\
78,91,114,115,123,139,140 \\
142,150,181,182,198\end{array}$ \\
\hline 210129 & $\begin{array}{c}8438 \\
25 \mathrm{Peg}\end{array}$ & B6-7Vne & 0.317 & $\begin{array}{l}5.71 \\
0.06\end{array}$ & $\begin{array}{l}0.283 \\
0.031\end{array}$ & $\begin{array}{l}0.96 \\
0.02\end{array}$ & $39316-43555$ & 13 & $\begin{array}{l}19,33,39,110,114,152,165 \\
169,198\end{array}$ \\
\hline 217050 & $\begin{array}{c}8731 \\
\text { EW Lac }\end{array}$ & B3IIIe ${ }^{a}$-shell & $0.165^{a}$ & $\begin{array}{l}5.31 \\
0.05\end{array}$ & $\begin{array}{l}0.231 \\
0.041\end{array}$ & $\begin{array}{l}0.96 \\
0.04\end{array}$ & $35400-43796$ & 419 & $2,38,80,85,91,110,142,198$ \\
\hline 217543 & 8758 & $\mathrm{~B} 3-4 \mathrm{IV}-\mathrm{Ve}^{a}$ & $0.210^{a}$ & $\begin{array}{l}6.53 \\
0.02\end{array}$ & $\begin{array}{l}0.183 \\
0.013\end{array}$ & $\begin{array}{l}0.90 \\
0.03\end{array}$ & $34348-43373$ & 51 & $38,85,86,91,110,142,198$ \\
\hline 218674 & KY And & B3IVeShell & 0.186 & $\begin{array}{l}6.76 \\
0.02\end{array}$ & $\begin{array}{l}0.212 \\
0.011\end{array}$ & $\begin{array}{l}1.09 \\
0.02\end{array}$ & $34348-47028$ & 198 & $85,86,91,110,160,198$ \\
\hline
\end{tabular}


Table 1. continued

Notes: $D_{*}$ is the stellar $B D ;\left(\bar{V}, \bar{D}, \overline{\Phi_{\mathrm{rb}}}\right)$ and $\left(\sigma_{V}, \sigma_{D}, \sigma_{\Phi}\right)$ are the mean values of all found magnitudes $V, B D$, visible gradients and the respective dispersions; $\mathrm{JD}_{\mathrm{f}}-\mathrm{JD}_{\mathrm{l}}$ are $\mathrm{JD}-2400000$ of the first and last observation; $N$ is the number of individual triads $\left(V, D, \Phi_{\mathrm{rb}}\right)$; " a " stands for $B C D$ classification and for $B C D$ values of $D_{*}$.

References:

1 Adelman (1992)

2 Alvarez \& Schuster (1982)

3 Appenzeller (1966)

5 Baldinelli et al. (1981)

6 Balona et al. (1992)

7 Barbier (1947)

8 Barrera et al. (1991)

9 Barylak \& Doazan (1986)

10 Belyakina \& Chugainov (1960)

11 Böhme (1984)

12 Böhme (1985)

13 Böhme (1986)

14 Böhme (1988)

15 Bouigue (1959)

16 Božič et al. (1995)

17 Brodskaja (1968)

18 Brucato \& Kristian (1972)

19 Cameron (1966)

20 Catalano \& Umana (1987)

21 Chochol et al. (1985)

22 Clariá \& Escosteguy (1981)

23 Corben (1966)

24 Corben (1971)

25 Cousins (1962)

26 Cousins \& Stoy (1963)

27 Cousins (1964)

28 Cousins (1965)

$\begin{array}{ll}28 & \text { Cousins }(1965) \\ 29 & \text { Cousins (1973) }\end{array}$

30 Cousins \& Stoy (1962)

31 Cousins \& Stoy (1970)

32 Crawford (1963a)

33 Crawford (1963b)

34 Crawford \& Barnes (1970)

35 Crawford et al. (1966)

36 Crawford et al. (1970)

37 Crawford et al. (1971a)

38 Crawford et al. (1971b)

39 Crawford et al. (1973)

40 Dahn \& Gueter (1973)

41 Danks \& Houziaux (1978)

42 Dapergolas et al. (1981)

43 de Loore et al. (1979)

44 Denoyelle (1977)

45 Deutschman et al. (1976)

46 Divan \& Zorec (1982b)

47 Divan \& Zorec (1982c)

48 Divan et al. (1983)

49 Doazan et al. (1982a)

50 Echevarría et al. (1979)

51 Eggen (1955)

52 Eggen (1963a)

53 Eggen (1963b)

54 Eggen (1968)

55 Elliot (1974)

56 Erro (1969)

57 Feinstein (1967)

$\begin{array}{ll}58 & \text { Feinstein }(1968 \mathrm{a}) \\ 59 & \text { Feinstein }(1968 \mathrm{~b})\end{array}$

60 Feinstein (1970)

61 Feinstein (1974)

62 Feinstein (1975)

63 Feinstein (1980)

64 Feinstein \& Marraco (1979)

65 Fernie (1991)

66 Ferrari-Toniolo et al. (1977)
67 Ferrari-Toniolo et al. (1978)

68 Franco (1989)

69 Frohlich \& Nevo (1974)

70 Garrison \& Kormendy (1976)

71 Golay (1958)

72 Gray \& Olsen (1991)

73 Grønbech \& Olsen (1976)

74 Guetter (1974)

75 Gulliver A.F. (1983)

76 Guo et al. (1995)

77 Gutierrez-Moreno \& Moreno (1968)

78 Gutierrez-Moreno et al. (1966)

79 Häggkvist (1971)

80 Häggkvist \& Oja (1966)

81 Häggkvist \& Oja (1969)

82 Häggkvist \& Oja (1970)

83 Hardie \& Crawford (1961)

84 Harmanec et al. (1978)

85 Harmanec et al. (1980)

86 Harris (1955)

87 Harris (1956)

88 Haug (1970)

89 Haupt (1974)

90 Haupt \& Moffat (1973)

91 Haupt \& Schroll (1974)

92 Heck \& Manfroid (1980)

93 Hill (1970)

94 Hiltner (1956)

95 Hiltner \& Johnson (1956)

96 Hirata (1995)

97 Hoag et al. (1961)

98 Hogg (1958)

99 Hopp \& Witzigmann (1980)

100 Hopp et al. (1982)

101 Horaguchi et al. (1994)

102 Horn et al. (1996)

103 Horn et al. (1982)

104 Howarth (1979)

105 Hutchings (1977)

106 Hutchison (1974)

107 Iliev et al. (1991)

108 Iriarte (1965)

109 Iriarte et al. (1965)

110 Jaschek et al. (1980)

111 Jeong \& Lee (1988)

112 Johnson (1964)

113 Johnson \& Harris (1954)

114 Johnson et al. (1966)

115 Johnson et al. (1967)

116 Johnson \& Morgan (1953)

117 Juza et al. (1994)

118 Kalv (1977)

119 Kiehling (1984)

120 Kilkenny et al. (1985)

121 Kozok (1984)

122 Kozok (1985)

123 Lake (1965)

124 Landolt (1969)

125 Landolt (1971)

126 Lee (1968)

127 Lenouvel \& Daguillon (1956)

128 Lesh (1968)

129 Lindroos (1983)

130 Ljunggren \& Oja (1964)

131 Loden (1969)

132 Lucke (1974)
133 Lutz \& Lutz (1972)

134 Lutz \& Lutz (1977)

135 Lynas-Gray \& Hill (1979)

136 Lynga (1959)

137 McNamara (1976)

138 Malmquist et al. (1960)

139 Manfroid et al. (1991)

140 Manfroid et al. (1995)

141 Margon et al. (1976)

142 Mendoza (1958)

143 Menzies et al. (1990)

144 Mitchell (1960)

145 Moffet \& Barnes (1979a)

146 Moffet \& Barnes (1979b)

147 Mook et al. (1974)

148 Moreno (1971)

149 Nakagiri \& Hirata (1979)

150 Oblak \& Chareton (1980)

151 Oja (1991)

152 Osawa \& Hata (1960)

153 Osawa \& Hata (1962)

154 Papousek (1979)

155 Pavlovski et al. (1996)

156 Pavlovski \& Ružič (1990)

157 Penprase (1992)

158 Penston (1973)

159 Percy (1986)

160 Percy et al. (1988)

161 Pfleiderer et al. (1966)

162 Philip \& Philip (1973)

163 Poretti (1982)

164 Roche et al. (1993)

165 Roman (1955)

166 Rucinski (1987)

167 Salukvadze \& Javakhishvili (1995)

168 Schneider (1987)

169 Schuster \& Alvarez (1983)

170 Schuster \& Guichard (1984)

171 Searle (1958)

172 Sharov \& Lyutyi (1988)

173 Sharov \& Lyutyi (1992)

174 Sharpless (1952)

175 Shobbrook (1984)

176 Simonson (1968)

177 Slawson et al. (1992)

178 Sowell \& Wilson (1993)

179 Stagg (1987)

180 Štefl et al. (1995)

181 Sterken et al. (1993)

182 Sterken et al. (1995)

183 Stokes (1972)

184 Stoy (1963)

185 Stoy $(1968)$

186 Tempesti \& Patriarca (1976)

187 Turner (1976)

188 van der Wal et al. (1972)

189 Vogt (1976)

190 Warman \& Echevarría (1977)

191 Warren \& Hesser (1978)

192 Westerlund (1963)

193 Westin (1982)

194 Wiegandt (1984)

195 Zelwanowa \& Schoneich (1971)

196 Zorec (1986)

197 Zorec (1994)

198 Geneva Obs. photom. archives 
Table 2. Stars used to represent the spectrophotometric parameters $D$ and $\Phi_{\mathrm{rb}}$ as functions of photometric indices

\begin{tabular}{|c|c|c|c|c|c|c|c|c|c|c|c|}
\hline HD & $\mathrm{HR}$ & Sp.Type & $\begin{array}{c}D \\
\operatorname{dex}\end{array}$ & $\begin{array}{l}\Phi_{\mathrm{rb}} \\
\mu \mathrm{m}\end{array}$ & Notes & HD & $\mathrm{HR}$ & Sp.Type & $\begin{array}{c}D \\
\operatorname{dex}\end{array}$ & $\begin{array}{l}\Phi_{\mathrm{rb}} \\
\mu \mathrm{m}\end{array}$ & Notes \\
\hline 886 & 39 & B2IV & 0.146 & 0.680 & 3,4 & 40111 & 2084 & B1Ib & 0.062 & 1.070 & \\
\hline 3360 & 153 & B2IV & 0.146 & 0.760 & $3,4,7$ & 40589 & 2111 & B9Iab & 0.266 & 1.580 & \\
\hline 4142 & 189 & $\mathrm{~B} 5 \mathrm{~V}$ & 0.272 & 0.760 & 2,7 & 41753 & 2159 & B3-4V & 0.217 & 0.790 & 7 \\
\hline 11241 & 533 & $\mathrm{~B} 2 \mathrm{~V}$ & 0.144 & 0.840 & 1,7 & 43112 & 2222 & B1IV & 0.096 & 0.700 & 1,7 \\
\hline 16582 & 779 & B2III & 0.136 & 0.760 & 3 & 43384 & 2240 & B4Ia & 0.107 & 1.940 & \\
\hline 19356 & 936 & $\mathrm{~B} 7-8 \mathrm{~V}$ & 0.350 & 0.930 & & 44743 & 2294 & B1II-III & 0.089 & 0.729 & 4 \\
\hline 20365 & 987 & B4V & 0.234 & 1.010 & & 45563 & 2347 & B9V & 0.422 & 0.839 & \\
\hline 20418 & 989 & B4IV & 0.244 & 0.970 & 7 & 46075 & 2374 & B6IV-V & 0.316 & 0.920 & \\
\hline 21071 & 1029 & $\mathrm{~B} 5 \mathrm{~V}$ & 0.270 & 0.980 & 7 & 47240 & 2432 & B1Iab & 0.070 & 1.400 & 6 \\
\hline 21278 & 1034 & $\mathrm{~B} 4-5 \mathrm{~V}$ & 0.249 & 0.980 & $2,4,7$ & 47432 & 2442 & O8Ia & 0.021 & 1.390 & 1,6 \\
\hline 21428 & 1044 & $\mathrm{~B} 4 \mathrm{~V}$ & 0.235 & 0.940 & 7 & 47756 & 2454 & B5-6IV & 0.292 & 0.920 & \\
\hline 21856 & 1074 & B1IV-V & 0.094 & 1.040 & 2,7 & 47964 & 2461 & B7III & 0.340 & 1.030 & \\
\hline 22928 & 1122 & B5-6III & 0.286 & 0.880 & 3,7 & 48434 & 2479 & O9.5Ib & 0.042 & 1.110 & 7 \\
\hline 22951 & 1123 & $\mathrm{~B} 0.5 \mathrm{~V}$ & 0.089 & 1.090 & 5 & 48977 & 2494 & B3V & 0.196 & 0.800 & \\
\hline 23288 & 1140 & $\mathrm{~B} 7 \mathrm{~V}$ & 0.340 & 1.080 & 4,5 & 49567 & 2517 & B4II-III & 0.195 & 0.870 & \\
\hline 23324 & 1144 & B7V & 0.342 & 1.060 & $2,4,7$ & 71155 & 3314 & A1V & 0.487 & 0.808 & $2,3,4,5,7$ \\
\hline 23338 & 1145 & B6IV & 0.297 & 0.970 & 3,4 & 74280 & 3454 & B3V & 0.181 & 0.730 & $3,4,5,7$ \\
\hline 23408 & 1149 & B6-7III & 0.315 & 1.030 & & 83754 & 3849 & B5V & 0.250 & 0.870 & $3,4,7$ \\
\hline 23432 & 1151 & B8IV-V & 0.376 & 1.060 & 4 & 87901 & 3982 & B7IV & 0.348 & 0.762 & $3,4,5,6,7$ \\
\hline 23753 & 1172 & B7V & 0.352 & 1.070 & 4,7 & 100600 & 4456 & B3-4V & 0.212 & 0.820 & 4 \\
\hline 23850 & 1178 & B7III & 0.340 & 0.980 & 3,5 & 120315 & 5191 & $\mathrm{~B} 4 \mathrm{~V}$ & 0.222 & 0.750 & $4,5,7$ \\
\hline 23923 & 1183 & B9V & 0.413 & 0.960 & 4 & 147394 & 6092 & B5IV-V & 0.265 & 0.850 & $2,4,7$ \\
\hline 24131 & 1191 & $\mathrm{~B} 1 \mathrm{~V}$ & 0.100 & 1.150 & & 160762 & 6588 & B3IV & 0.204 & 0.750 & $4,5,7$ \\
\hline 24640 & 1215 & $\mathrm{~B} 2 \mathrm{~V}$ & 0.145 & 1.010 & & 166182 & 6787 & B2.5III & 0.156 & 0.830 & 2,7 \\
\hline 24760 & 1220 & B0III & 0.075 & 0.760 & $3,5,7$ & 184915 & 7446 & B1II & 0.076 & 1.040 & $4,6,7$ \\
\hline 25638 & 1260 & B0III & 0.059 & 1.940 & & 191692 & 7710 & A0IV & 0.502 & 0.820 & 6 \\
\hline 30211 & 1520 & B5IV & 0.251 & 0.790 & 7 & 202850 & 8143 & B9Ia & 0.240 & 0.981 & 4,7 \\
\hline 32630 & 1641 & $\mathrm{~B} 4 \mathrm{~V}$ & 0.230 & 0.780 & $1,3,4,7$ & 204172 & 8209 & B0Ib & 0.048 & 1.010 & 7 \\
\hline 35468 & 1790 & B2III & 0.132 & 0.710 & $1,3,7$ & 205021 & 8238 & B1III-IV & 0.095 & 0.710 & \\
\hline 35600 & 1804 & B9Ib & 0.363 & 1.490 & & 206165 & 8279 & B2.5Ib & 0.108 & 1.620 & 4,7 \\
\hline 36371 & 1843 & B4Ia & 0.110 & 1.790 & & 208218 & & B2II-III & 0.110 & 1.520 & 2 \\
\hline 36959 & 1886 & $\mathrm{~B} 1 \mathrm{~V}$ & 0.115 & 0.730 & & 208440 & & B1IV & 0.091 & 1.210 & 2 \\
\hline 36960 & 1887 & B0IV & 0.072 & 0.720 & 1,7 & 209339 & 8399 & O9IV & 0.052 & 1.210 & 7 \\
\hline 37016 & 1891 & B3-4V & 0.196 & 0.840 & & 209961 & 8427 & B2III-IV & 0.156 & 0.835 & \\
\hline 37040 & 1898 & B3V & 0.184 & 0.860 & & 212978 & 8553 & B2III & 0.150 & 0.850 & 5 \\
\hline 37043 & 1899 & O7III-IV & 0.035 & 0.700 & 3,6 & 214240 & 8606 & B4-5III & 0.252 & 0.848 & 2 \\
\hline 37209 & 1911 & $\mathrm{~B} 1 \mathrm{~V}$ & 0.109 & 0.760 & 7 & 214680 & 8622 & O8:V & 0.048 & 0.790 & $1,2,4,5$ \\
\hline 37468 & 1931 & $\mathrm{O} 9 \mathrm{~V}$ & 0.057 & 0.700 & 6 & 217101 & 8733 & $\mathrm{~B} 2 \mathrm{~V}$ & 0.132 & 0.780 & 2,7 \\
\hline 37481 & 1933 & $\mathrm{~B} 1 \mathrm{~V}$ & 0.116 & 0.750 & 7 & 218376 & 8797 & B0.5II & 0.074 & 1.050 & 1,7 \\
\hline 37744 & 1950 & $\mathrm{~B} 1.5 \mathrm{~V}$ & 0.124 & 0.790 & 7 & 218407 & 8800 & $\mathrm{~B} 2 \mathrm{~V}$ & 0.165 & 0.845 & 2 \\
\hline 38666 & 1996 & O8-9V & 0.050 & 0.700 & 6 & 222173 & 8965 & B8IV & 0.380 & 0.833 & $2,3,4,5,7$ \\
\hline
\end{tabular}

$U B V$ data of comparison stars were taken from Mermilliod \& Mermilliod's (1994) compilation. The relation giving the $B D$ from $Q_{U B V}$ is then:

$D_{U B V}=(0.507 \pm 0.008) Q_{U B V}+(0.496 \pm 0.005) \operatorname{dex} .(2)$

Figure 1a shows $D_{B C D}$ versus $Q_{U B V}$. Relation (2) has a linear correlation coefficient $r=0.992$ and leads to a mean
$(\mathrm{O}-\mathrm{C})$ dispersion $\sigma_{(\mathrm{O}-\mathrm{C})}=0.014$ dex. The gradient $\Phi_{\mathrm{rb}}$ is obtained using the $(B-V)$ colour index as:

$\Phi_{\mathrm{rb}(U B V)}=(1.80 \pm 0.11)(B-V)+(1.11 \pm 0.02) \quad \mu \mathrm{m} .(3)$

The correlation between $\Phi_{\operatorname{rb}(U B V)}$ and $\Phi_{\operatorname{rb}(B C D)}$ is given by:

$\Phi_{\mathrm{rb}(U B V)}=(1.00 \pm 0.11) \Phi_{\mathrm{rb}(B C D)}+(0.00 \pm 0.10) \quad \mu \mathrm{m}(4)$ 
with $r=0.975$ and $\sigma_{(\mathrm{O}-\mathrm{C})}=0.06 \mu \mathrm{m}$. Figure $1 \mathrm{~b}$ shows the correlation between the $\Phi_{\mathrm{rb}(B C D)}$ gradient and the $(B-V)$ index.

\section{ii) The $U B V R I$ system.}

The $B D$ is obtained from relation (2) as for data in the $U B V$ system. The gradient $\Phi_{\mathrm{rb}}$ is now defined by also taking into account the $(V-R)$ colour index, in order to better respect the wavelength interval over which the original $\Phi_{\mathrm{rb}(B C D)}$ gradient is defined. However, with the inclusion of the $(V-R)$ index we cannot avoid introducing a new source of error. The resulting relation is:

$$
\begin{aligned}
\Phi_{\mathrm{rb}(U B V R I)=} & (1.63 \pm 0.02)(B-V)+(0.39 \pm 0.11) \\
& (V-R)+(1.10 \pm 0.09) \mu \mathrm{m} .
\end{aligned}
$$

The correlation between $\Phi_{\mathrm{rb}(U B V R I)}$ and $\Phi_{\mathrm{rb}(B C D)}$ is:

$\Phi_{\mathrm{rb}(U B V R I)}=(0.94 \pm 0.05) \Phi_{\mathrm{rb}(B C D)}+(0.06 \pm 0.05) \mu \mathrm{m}(6)$ with $r=0.968$ and $\sigma_{(\mathrm{O}-\mathrm{C})}=0.07 \mu \mathrm{m}$. Gradients $\Phi_{\mathrm{rb}(U B V R I)}$ against $\Phi_{\mathrm{rb}(B C D)}$ are shown in Figure 1. The $U B V R I$ photometric data used to derive relation (5) are from the "Photoelectric Photometric Catalogue in the Johnson UBVRI System" (Lanz 1986).

iii) The Strömgren uvby system.

As for the $U B V$ system, we derive the value of the $B D$ by defining an ISM reddening-free Johnson's like index $Q$ in the Strömgren uvby system. This index was determined using the mean ISM absorption law of Mathis (1990) to determine the colour excess factor $E(u-v) / E(b-y)$ :

$Q_{u v b y}=(u-v)-1.079(b-y) \quad$ mag.

The $B D$ is then given by:

$D_{u v b y}=(0.349 \pm 0.006) Q_{u v b y}+(0.070 \pm 0.003) \operatorname{dex}$

with $r=0.997$ and $\sigma_{(\mathrm{O}-\mathrm{C})}=0.009$ dex. The $B D$ could also be derived using the $c_{1}$ index of the uvby system by:

$D_{\text {uvby }}=(0.383 \pm 0.007) c_{1}+(0.086 \pm 0.002) \operatorname{dex}$

but $r=0.986$ and $\sigma_{(\mathrm{O}-\mathrm{C})}=0.014$ dex. In our work we used relation (8). The gradient $\Phi_{\mathrm{rb}}$ is obtained from:

$$
\begin{aligned}
\Phi_{\mathrm{rb}(\text { uvby })=} & (1.28 \pm 0.20)(v-b)+(1.61 \pm 0.13)(b-y) \\
& +(0.91 \pm 0.01) \mu \mathrm{m} .
\end{aligned}
$$

The correlation between $\Phi_{\mathrm{rb}(u v b y)}$ and $\Phi_{\mathrm{rb}(B C D)}$ is:

$\Phi_{\mathrm{rb}(u v b y)}=(0.97 \pm 0.05) \Phi_{\mathrm{rb}(B C D)}+(0.03 \pm 0.05) \mu \mathrm{m}(11)$

where $r=0.986$ and $\sigma_{(\mathrm{O}-\mathrm{C})}=0.04 \mu \mathrm{m}$. In Fig. 3a we show the relation between $D_{u v b y}$ and $Q_{u v b y}$, and in Fig. 3b the relation between $\Phi_{\mathrm{rb}(u v b y)}$ and $\Phi_{\mathrm{rb}(B C D)}$. Relations (8) to (10) were derived using data given in the $u v b y-\beta$ Photoelectric Photometric Catalogue (distributed by the
CDS) (Hauck \& Mermilliod 1975; Grønbech \& Olsen 1976, 1977).

$i v)$ Thirteen-colour photometry.

As in preceding cases, we also defined an ISM reddeningfree index $Q_{13 \mathrm{c}}$ using the $\left(m_{35}-m_{40}\right)$ and $\left(m_{45}-m_{52}\right)$ colour indices, where $m_{35}, m_{40}, m_{45}$ and $m_{52}$ are magnitudes respectively at $0.3536,0.4030,0.4571$ and 0.5183 $\mu \mathrm{m}$ effective wavelengths of the 13-colour medium-narrowband photometric system (Johnson \& Mitchell 1975). The $Q_{13 c}$ index is defined as:

$Q_{13 c}=\left(m_{35}-m_{40}\right)-0.995\left(m_{45}-m_{52}\right) \quad$ mag.

The resulting relation for the $B D$ is:

$D_{13 \mathrm{c}}=(0.382 \pm 0.008) Q_{13 \mathrm{c}}+(0.499 \pm 0.007) \quad \operatorname{dex}$

where $r=0.994$ and $\sigma_{(\mathrm{O}-\mathrm{C})}=0.013$ dex. For the gradient $\Phi_{\mathrm{rb}}$ the relation is:

$\begin{aligned} \Phi_{\mathrm{rb}(13 \mathrm{c})}= & (1.43 \pm 0.28)\left(m_{45}-m_{52}\right)+(1.26 \pm 0.20) \\ & \left(m_{52}-m_{62}\right)+(0.99 \pm 0.03) .\end{aligned}$

The relation between $\Phi_{\mathrm{rb}(13 \mathrm{c})}$ and $\Phi_{\mathrm{rb}(B C D)}$ is given by:

$\Phi_{\mathrm{rb}(13 \mathrm{c})}=(0.95 \pm 0.06) \Phi_{\mathrm{rb}(B C D)}+(0.05 \pm 0.06) \mu \mathrm{m}(15)$

for which $r=0.977$ and $\sigma_{(\mathrm{O}-\mathrm{C})}=0.06 \mu \mathrm{m}$. In the $13-$ colour photometric system the $V$ magnitude was obtained as:

$$
\begin{aligned}
V= & (0.528 \pm 0.002) m_{52}+(0.469 \pm 0.002) m_{58} \\
& +(0.002 \pm 0.010) \mathrm{mag}
\end{aligned}
$$

whose comparison with the original $V$ magnitude of the $U B V$ system has a correlation coefficient $r=0.999$ and $\sigma_{(\mathrm{O}-\mathrm{C})}=0.030 \mathrm{mag}$. Figure $4 \mathrm{a}$ shows the relation between $D_{13 \mathrm{c}}$ and $Q_{u v b y}$, Fig. $4 \mathrm{~b}$ that between $\Phi_{\mathrm{rb}(13 \mathrm{c})}$ and $\Phi_{\mathrm{rb}(B C D)}$ and Fig. 4c that between $V$ and $V_{13 \mathrm{c}}$.

$v)$ The Geneva photometric system.

The ISM reddening-free $Q$ index corresponding to this system was defined as:

$Q_{\mathrm{G}}=\left(U-B_{1}\right)-1.48\left(B_{1}-B_{2}\right) \mathrm{mag}$

from which the respective $B D$ can be derived as:

$D_{\mathrm{G}}=(0.482 \pm 0.007) Q_{\mathrm{G}}-(0.267 \pm 0.007) \operatorname{dex}$

where $r=0.985$ and $\sigma_{(\mathrm{O}-\mathrm{C})}=0.019$ dex. It can also be shown that:

$D_{\mathrm{G}}=(0.473 \pm 0.024) d-(0.243 \pm 0.022) \operatorname{dex}$

where $d$ is a measure of the $B D$ defined in the Geneva photometric system (Rufener 1981); here we have $r=0.978$ 
and $\sigma_{(\mathrm{O}-\mathrm{C})}=0.024 \mathrm{dex}$. In our work we used the value $D_{\mathrm{G}}$ given by (18). The gradient $\Phi_{\mathrm{rb}}$ is defined as:

$$
\begin{aligned}
\Phi_{\mathrm{rb}(\mathrm{G})=} & (0.44 \pm 0.08)\left(B_{2}-V_{1}\right)+(1.15 \pm 0.06) \\
& \left(B_{2}-G\right)+(1.83 \pm 0.03) \mu \mathrm{m}
\end{aligned}
$$

which compares with $\Phi_{\mathrm{rb}(B C D)}$ as:

$\Phi_{\mathrm{rb}(\mathrm{G})}=(0.93 \pm 0.04) \Phi_{\mathrm{rb}(B C D)}+(0.07 \pm 0.04) \quad \mu \mathrm{m}$

so that $r=0.964$ and $\sigma_{(\mathrm{O}-\mathrm{C})}=0.07 \mu \mathrm{m}$. Data for comparison stars concerning the Geneva photometric system were taken from Rufener $(1980,1988)$. Figure 5a shows the relation between $D_{\mathrm{G}}$ and $Q_{\mathrm{G}}$ and Figure $5 \mathrm{~b}$ that between $\Phi_{\mathrm{rb}(\mathrm{G})}$ and $\Phi_{\mathrm{rb}(B C D)}$.

\subsection{Uncertainties affecting the $\Phi_{\mathrm{rb}}$ and $D$ parameters}

BDs of Be stars in the $B C D$ system are given with typical errors $\delta D_{*} \simeq 0.015$ dex. The width of a $D_{*}$ interval of a given spectral type-box in the $B C D$ system depends on the effective temperature and on the luminosity class. The mean value of these widths is $\Delta D_{*}=0.040 \pm 0.008 \mathrm{dex}$ (Chalonge \& Divan 1973). Comparing the $B C D$ spectral type determinations of program stars with those given in the MK system for the same objects (Slettebak 1982) we derived the mean absolute spectral type deviation between $B C D$ and $\mathrm{MK}$ determinations $\Delta$ (Sp.T.) $=0.5 \pm 0.4$ (of sub-spectral type). Hence, we can argue that for those Be stars where only MK classification exists, the value of $D_{*}$ adopted in Table 1 may be in error by $\delta D_{*} \lesssim 0.04$ dex.

Among the most important uncertainties affecting the indirect $\Phi_{\mathrm{rb}}$ and $D$ parameters derived from relations given in Sect 2.5.2 are: (1) random uncertainties of original photometric data; (2) systematic differences related to the effective wavelengths of photometric magnitudes; (3) presence of variable spectral lines in the filter wavelength pass-bands; (4) extrapolations of $D$ values in phases of strong emission in the second $B D$ component.

(1) - Uncertainties related to photometric measurements are difficult to determine, as it is not easy to compare measurements given in different systems or even in the various versions of the same photometric system (Sterken \& Manfroid 1992). Using the mean errors of individual measurements of colour indices in different photometric systems (CETAMA 1978; Crawford \& Barnes 1970; Johnson et al. 1967; Manfroid et al. 1991; Rufener 1981; Shuster 1976; Sterken \& Manfroid 1992) and the relations between $D, \Phi_{\mathrm{rb}}$ and the concerned colour indices given in Sect. 2.5.2, we derived the expected mean errors of individual $D$ and $\Phi_{\mathrm{rb}}$ for each photometric system used in this work. They are given in Table 3. Whenever possible, we checked that the photometric indices of B0-A1 comparison and/or check stars in the various photometric systems used or versions of same systems did not introduce systematic scatterings higher than those found when establishing the transformation relations of Sect. 2.5.2. It
Table 3. Mean errors of individual $\Phi_{\mathrm{rb}}$ and $D$

\begin{tabular}{ccc}
\hline System & $\begin{array}{c}\epsilon(\Phi) \\
\mu \mathrm{m}\end{array}$ & $\begin{array}{c}\epsilon(D) \\
\operatorname{dex}\end{array}$ \\
\hline $\begin{array}{c}\text { BCD } \\
\text { Genève }\end{array}$ & 0.035 & 0.015 \\
uvby & 0.070 & 0.020 \\
$13-\mathrm{c}$ & 0.085 & 0.020 \\
$U B V$ & 0.054 & 0.026 \\
$U B V R I$ & 0.075 & 0.026 \\
\hline
\end{tabular}

is however known, mainly for the $(U-B)$ colour indices, that according to different authors high systematic deviations may exist. When such deviations were detected, the data concerned were discarded. Systematic differences are sometimes found among uvby photometric indices if the filters used are not the same. By comparing non variable stars observed in these slightly different systems and/or looking for simultaneous observations of the studied $\mathrm{Be}$ stars, we reduced such observations to a common frame. When these reductions were not possible we did no use the existent data.

(2) - The strongest emission in the Balmer continuum appears at $\lambda 0.365 \mu \mathrm{m}$. As the filter-detector response in near-UV is frequently only a few per cent at this wavelength, the reddening effect induced by the $\mathrm{CE}$ continuum emission near the $B D$ (scaled by calibrations based on normal B stars of Sect. 2.5) can then be underestimated. Hence, relations like (8) or (9) of the uvby system and (13) in the Thirteen-colour photometry may in principle give slightly underestimated values of the total $B D$.

(3) - Variations of the $\left(\Phi_{\mathrm{rb}}, D\right)$ parameters cannot always be attributed to variations of the continuum spectrum alone. Spurious reddening and bluening effects can be produced by variable CE emission/absorption components in lines entering the filter wavelength pass-bands. A very rough upper limit of the reddening effect produced by absorption lines in the $\left(\Phi_{\mathrm{rb}}, D\right)$ parameters can be estimated by comparing the $U B V$ colour indices of low and high line-blanketed models of stellar atmospheres (Kurucz et al. 1974; Kurucz 1992). Thus, the averaged systematic reddening effect found in the $(B-V)$ and $(U-B)$ indices for $\log g=2.0$ to 4.5 and for all effective temperatures from $T_{\text {eff }}=8000$ to $30000 \mathrm{~K}$ leads to a change $\Delta \Phi_{\mathrm{rb}}=0.09 \pm 0.04$. Similarly, the highest increase of the $B D, \Delta D=0.06 \pm 0.02 \mathrm{dex}$, is for $T_{\text {eff }}=10^{4} \mathrm{~K}$. It is however much lower for other effective temperatures, especially among the hottest ones. In actual Be stars, we may then expect that, depending on the relative degree of the line emission/absorption variability produced by the CE entering the photometric filter pass-bands, the $\left(\Phi_{\mathrm{rb}}\right.$, $D)$ will show reddening or blueing components which do 
not directly reflect variations of the continuum spectrum only.

(4) - As relations between spectrophotometric $D$ and the photometric colour indices were obtained using stars without emission, when $D \sim 0$ or $D<0$ they are necessarily extrapolated. We compared such extrapolated values of $D$ with those obtained in the $B C D$ for similar strong emission phases of HD 5394, HD 24534, HD 60848, HD 148184 and HD 200120 which can be seen in Fig. 6. However, the nice agreement between both determinations do not indicate the existence of any strong deviation.

The total amount of the quoted uncertainties is almost impossible to estimate, because they depend on the star and on its variation phase. However, using as reference the $\left(\Phi_{\mathrm{rb}}, D\right)$ relations derived from $B C D$ system measurements only [which are independent of uncertainties (1) to (4)] of strongly variable Be stars, we can obtain a rough estimate of the mean total amount of possible deviations which affect the indirect $\Phi_{\mathrm{rb}}$ and $D$ parameters derived from multicolour filter photometry. Comparing the mean deviations of data in each photometric system with the mean regression lines derived with genuine $B C D$ parameters, we obtain $\overline{\Delta D}=0.02 \mathrm{dex}$ and $\overline{\Delta \Phi_{\mathrm{rb}}}=0.050 \mu \mathrm{m}$.

\section{Results}

The spectrophotometric data gathered for all program stars are presented in Fig. 6 (available in electronic form). For each star there are three panels. The first shows the observed time variability of the $V, \Phi_{\mathrm{rb}}$ and $D$. The second panel shows the $(U, D),(V, D)$ and $\left(\Phi_{\mathrm{rb}}, D\right)$ correlations and the third the $\left(\Phi_{\mathrm{rb}}, V\right)$ correlation. The symbols used are: black dots for the $B C D$ data, crosses for $U B V$ data, stars for $U B V R I$, triangles for Geneva photometry, diamonds for the uvby system and squares for 13-colour data. The following comments for individual stars are divided into two subsections according to the degree of known spectrophotometric and spectroscopic variations.

\subsection{Spectrophotometric and spectroscopic behaviour of some well-studied Be stars}

This subsection deals with program Be stars, for which we have enough $B C D$ spectrophotometric observations to allow us: (a) to estimate the uncertainties discussed in Sect. 2.6 ; (b) to assign not only a degree of reliability to relations derived using only indirect $\left(V, \Phi_{\mathrm{rb}}, D\right)$ parameters, but also to judge the likelihood of scatters and more or less systematic deviations from the sketched mean relations. As for all these objects rather copious spectroscopic observations exist, in this section we also summarize their main spectroscopic variations over the last fifty years, more or less simultaneous to photometric data which are analyzed in this paper. The qualitative description of the correlated spectroscopic and spectrophotometric behaviours of these objects can be used as a reference for the remaining pro- gram stars, for which only spectrophotometric variations are presented. These stars are divided into two groups: (1) Be stars most commonly seen in Be phases: $\gamma$ Cas, X Per, $11 \mathrm{Cam}, \chi$ Oph and 59 Cyg; (2) Be stars most frequently observed in Be-shell phases: Pleione, 48 Lib and 88 Her.

\subsubsection{Stars most frequently seen in Be phases}

\section{$\gamma$ Cas (HD 5394)}

Observed since 1866, $\gamma$ Cas firstly showed a long Be phase of strong emission. Then, between 1932 and 1942, dramatic changes occurred in its spectrum; two strong Be phases, each followed by a strong shell phase, have been observed (Doazan et al. 1983 and references therein). In 1942, $\gamma$ Cas entered a quasi-normal B phase. Four years later, a subsequent Be phase increased slowly and irregularly. Weak secondary minima in emission were reported in 1956-1961 (Hubert-Delplace \& Hubert 1979 hereafter referred to as "Atlas"), end 1975, 1983-1984 (Horaguchi et al. 1994 and references therein) and in 19901991 (Peters 1990, 1991); a "veiling" effect of lines was seen from the end of 1963 to 1972 (Atlas). According to Horaguchi et al. (1994), H $\alpha$ intensity has oscillated with a time scale of several years. Long-term far UV and visual variability seems to be associated (Doazan et al. 1987).

It is interesting to note that the minima in the $\Phi_{\mathrm{rb}}$ gradient correspond to minima in emission strength and the maximum of $\Phi_{\mathrm{rb}}$ in 1966 to a veiling in line spectra. Furthermore the total $B D$ is strongly negative at the epochs of strong outbursts (1932-1942). Somewhat scattered but single $\left(\Phi_{\mathrm{rb}}, D\right)$ and $\left(\Phi_{\mathrm{rb}}, V\right)$ correlations with $\partial \Phi_{\mathrm{rb}} / \partial(D, V)<0$ slopes are present, although photometric and spectrophotometric data correspond to epochs before and after a number of $\mathrm{Be} \rightleftharpoons \mathrm{B}$-normal $\rightleftharpoons$ Be-shell phase transitions. We note, however, that the $(V, D)$ relation has two branches in $-0.025 \lesssim D \lesssim D_{*}$ dex. Let us finally note that the rapid photometric variability which is commonly associated with surface stellar activity is still debated for this object.

\section{$X$ Per (HD 24534)}

Variable but generally weak emission was noted in the spectrum of X Per before the end of the forties (Cowley et al. 1972). In 1951-1953, a strong emission phase phase with a "veiling" effect began; afterwards marked strengthening (outburst) of emission lines (H I, He I, Fe II, Si II) and of the "veiling" effect from 1957 to 1961 has been reported, with a maximum in 1961 (Cowley et al. 1972; Wackerling 1972, Atlas). In 1974 the emission decreased for 2 years, becoming bright again by the end of 1976. In 1978, only very weak emission was seen in $\mathrm{H} \alpha$, the spectrum was consistent with a quasi-normal O9-B0 star (de Loore et al. 1979). By the end of 1978, a new emission phase reappeared (Roche et al. 1993 and references therein). Still fairly bright in February 1990, the $\mathrm{H} \alpha$ line was seen converted to absorption in September 1990. A strong shell feature superposed on the photospheric component of $\mathrm{H} \alpha$ and He I 6678 in Nov., Dec. 1990 and Jan. 1991 was reported 

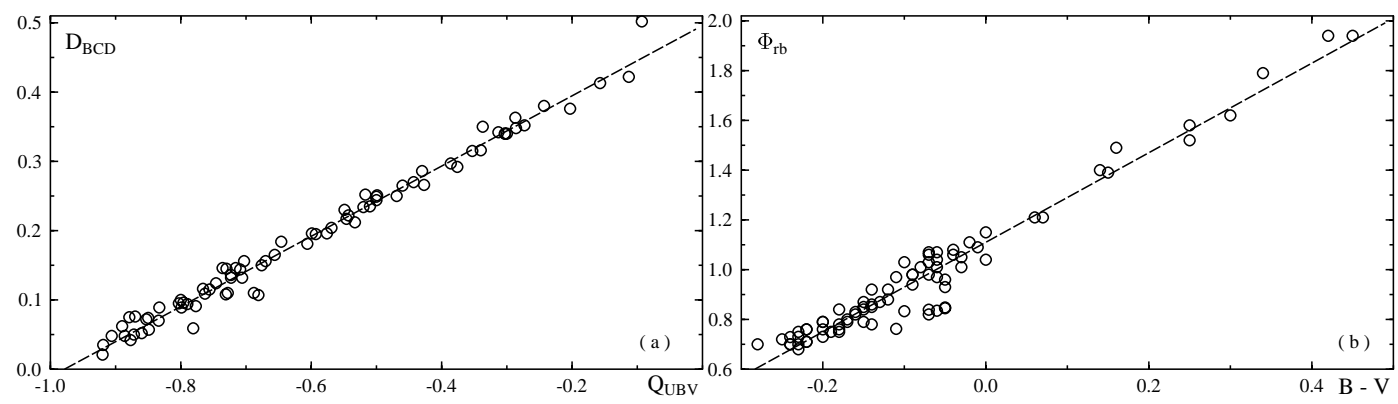

Fig. 1. a) Transformation of $Q_{U B V}$ to $D_{B C D}$. b) Transformation of the $(B-V)$ colour index to the gradient $\Phi_{\mathrm{rb}}$

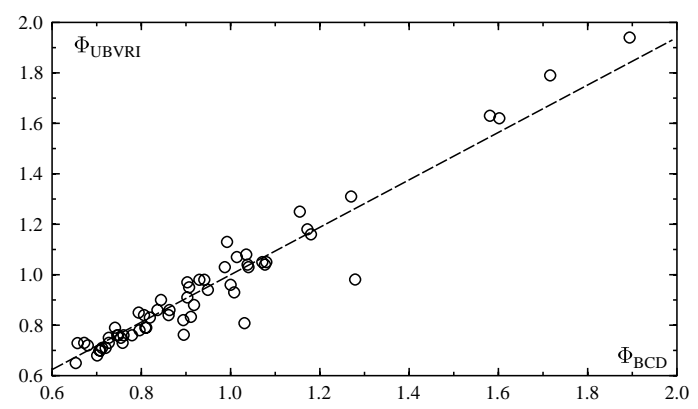

Fig. 2. Comparison of $\Phi_{\mathrm{rb}(U B V I R)}$ with $\Phi_{\mathrm{rb}(B C D)}$ gradients

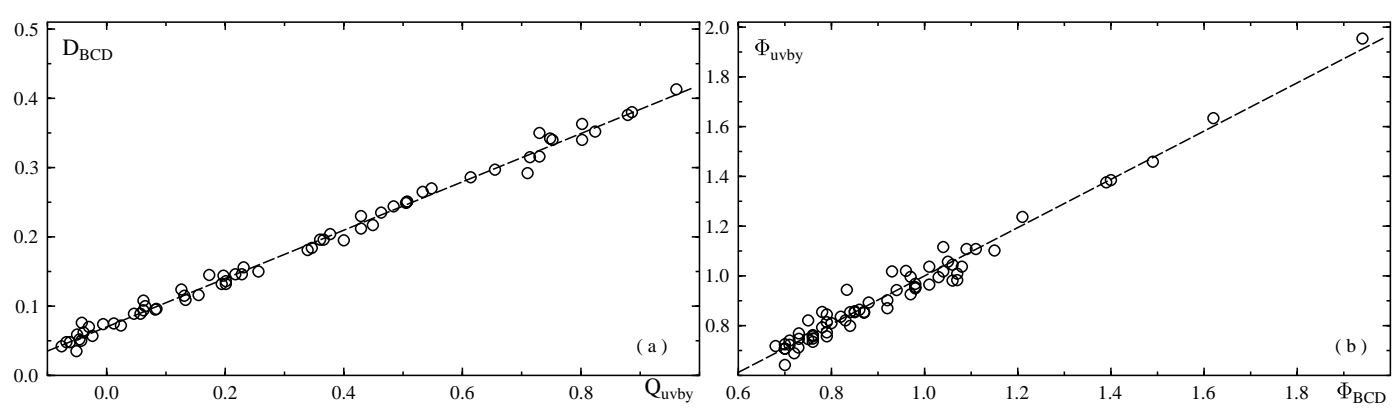

Fig. 3. a) Transformation of $Q_{\text {uvby }}$ to $D_{B C D}$. b) Comparison of $\Phi_{\operatorname{rb}(u v b y)}$ with $\Phi_{\operatorname{rb}(B C D)}$ gradients

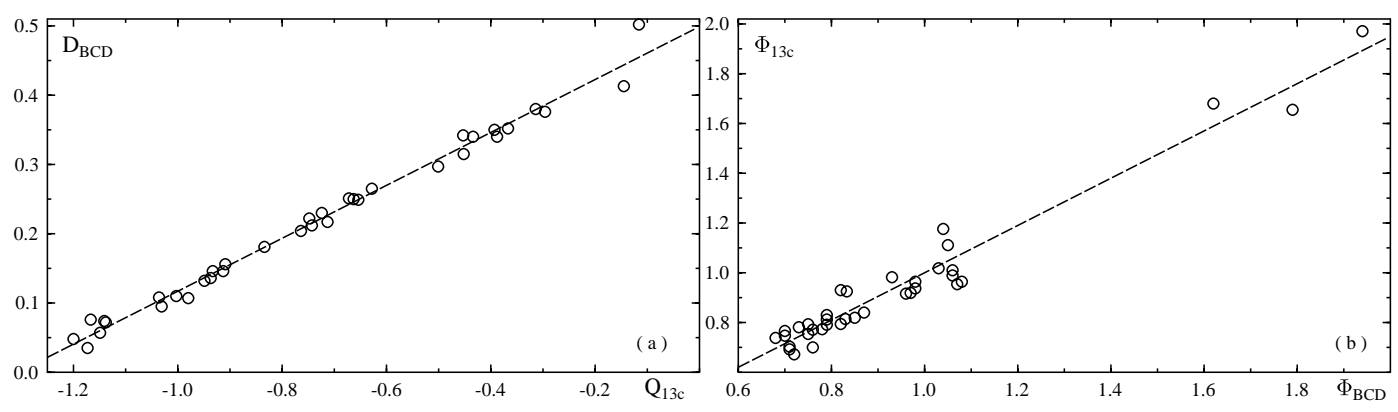

Fig. 4. a) Transformation of $Q_{13 \mathrm{c}}$ to $D_{B C D}$. b) Comparison of $\Phi_{13 \mathrm{c}}$ with $\Phi_{\mathrm{rb}(B C D)}$ gradients 


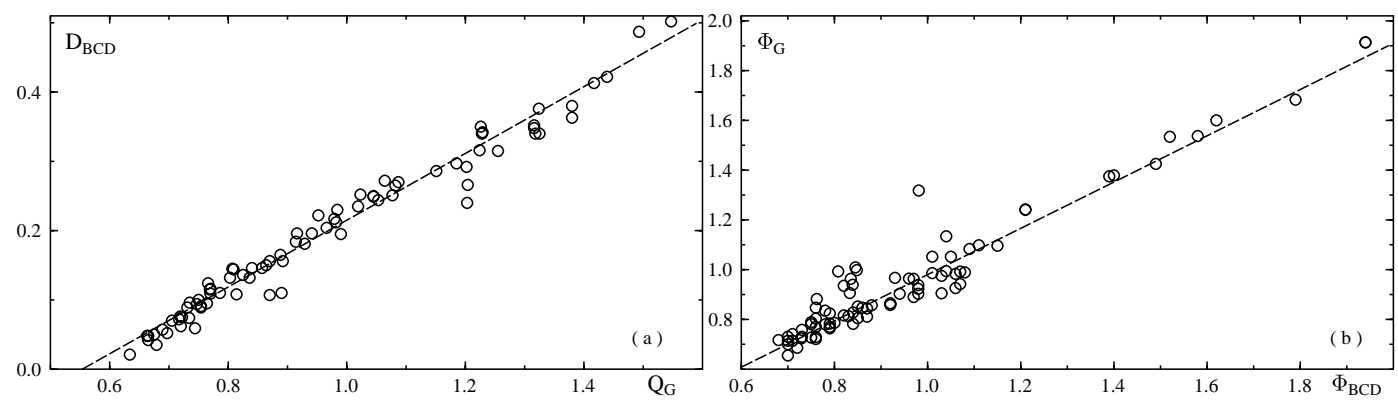

Fig. 5. a) Transformation of $Q_{\mathrm{G}}$ to $D_{B C D}$. b) Comparison of $\Phi_{\mathrm{rb}(\mathrm{G})}$ with $\Phi_{\mathrm{rb}(B C D)}$ gradients

by Norton et al. 1991. Still in absorption in Sept. 1991 (Reynolds et al. 1992), weak emission was again present on $\mathrm{H} \alpha$ in Oct. 1991 (Kaper \& van Kerkwijk 1992).

Decrease and minima of line emission generally correspond to lower brightness states. Single $\left(\Phi_{\mathrm{rb}}, D\right)$ and $\left(\Phi_{\mathrm{rb}}\right.$, $V)$ correlations are accompanied by bifurcated or doublefold $(U, D),(V, D)$ relations with $\partial(U, V) / \partial D>0$ slopes in $D<D_{*}$ corresponding to definite Be phases. The stellar $B D$ is $D_{*}=0.050$ dex (de Loore et al. 1979). For $D \gtrsim D_{*}$ incipient horizontal relations: $\partial\left(V, \Phi_{\mathrm{rb}}, U\right) / \partial D \simeq 0$ are apparent which correspond to the shell phase.

\section{Cam (HD 32343)}

11 Cam has always had strong emission observed up to high Balmer lines. Often observed before 1975, this star has presented recurrent outbursts. Schild (1973) reported outbursts in 1916-1919, 1929-1931 and 1943-1949. From 1953 to 1974, emission was always strong in Balmer lines with a weak minimum in 1961. Bright and particularly clear Fe II lines were reported from 1963 to 1971 during maximum emission. Afterwards they seem to have disappeared (Atlas). Isolated observations between 1980 and 1989 of the $\mathrm{H} \alpha$ emission line have revealed a rather constant strength of this line except in October 1983 (Ballereau \& Chauville 1987) for which the line is very strong (no photometry available at that time). Conversely no spectra are available at maximum brightness in November 1977. The rapid photometric variability of this star is suspected although not yet confirmed.

Single $(U, D),(V, D)$, and $\left(\Phi_{\mathrm{rb}}, V\right)$ relations are seen, although they all have $\partial(U, V) / \partial D<0$ and $\partial \Phi_{\mathrm{rb}} / \partial V>$ 0 slopes. The $\left(\Phi_{\mathrm{rb}}, D\right)$ relation is scattered and single, however it has a $\partial \Phi_{\mathrm{rb}} / \partial D<0$ slope.

\section{$\chi$ Oph (HD 148184)}

Since $1919 \chi$ Oph has been characterized by strong emission in Balmer lines (Schild 1973; Slettebak 1982). A very strong emission in Balmer and Fe II lines between 1953 and 1973 with a veiling effect seen up to 1969 (Atlas). From 1979, Fe II emission lines have decreased in intensity; they were barely visible in 1975. Andrillat \& Fehrenbach (1982) and Dachs et al. (1981) noted a steady decrease in the strength of $\mathrm{H} \alpha$ emission from 1972 to 1980. Then it seems to have slightly increased in 1982-1983, with a maximum through March 1983 (Dachs et al. 1986), and afterwards fluctuated with maxima $I / I_{\mathrm{c}}=10-11$ in 1985 , 1989 and 1993 (Hanuschik et al. 1996). The decrease of the $\mathrm{H} \alpha$ emission line from 1972 to 1980 corresponds to an increase of brightness and to reddening of the gradient $\Phi_{\mathrm{rb}}$. Total Balmer discontinuity $D$ varied strongly during this period with a strong minimum near 1983. $D$ is at minimum when $I / I_{\mathrm{c}}$ of $\mathrm{H} \alpha$ emission is at maximum. $\chi \mathrm{Oph}$ is a rapid photometric variable star (Balona 1995) with a moderate $V \sin i\left(<130 \mathrm{~km} \mathrm{~s}^{-1}\right)$.

The $(U, D)$ and $(V, D)$ relations are single for $D<$ 0 with $\partial(U, V) / \partial D<0$ slopes, but two branches with $\partial(U, V) / \partial D>0$ slopes seem to appear at $D \simeq 0$. The $\left(\Phi_{\mathrm{rb}}, D\right)$ and $\left(\Phi_{\mathrm{rb}}, V\right)$ relations are scattered and whilst the first is likely to be single with a $\partial \Phi_{\mathrm{rb}} / \partial D<0$ slope, the second seems to be double valued with one of the branches with a $\partial \Phi_{\mathrm{rb}} / \partial V>0$ slope.

\section{Cyg (HD 200120)}

Since 1904, 59 Cyg has presented active and quiescent phases in the behaviour of its $\mathrm{CE}$, successively producing spectra with the appearance of a quasi-normal B, Be and Be-shell star. Long-term, rather stable, strong emissionline phases alternate with phases of marked changes, generally characterized by slow increase and shorter decreases of the strength of emission lines. A slow increase of emission was observed from 1945 to 1950 (Merrill \& Burwell 1949). Afterwards, a quiescent phase set in from 1953 to 1970, the emission features being relatively stable; emission was at maximum in 1956 and in 1961, and at minimum in 1967 (Atlas). Short-lived outbursts were then observed from July to the end of 1972 and from December 1973 to June 1974, each followed by a strong shell phase of several months duration. After that, emission vanished and almost completely disappeared in 1976-1977 (HubertDelplace \& Hubert 1981). An increase of emission started in the end of 1979 and ended near mid-1980. Emission decreased again to a minimum in 1982, and again increased from 1983 to 1986 . From 1979 to 1985 strong $\mathrm{H} \alpha$ emission strength changes occurred, being correlated with the wind observed in the far UV (Doazan et al. 1989). From 1986, 
a new, rather stable quiescent emission phase has been established (Peters 1989-1992, 1994).

Strong emission lines are present when $D<D_{*}$ and the line emission decrease corresponds to a progressive increase of the $B D$, which ended at $D=D_{*}$ in 1977 when the line emission almost disappeared. From the existing photometric data, it is seen that after 1961 the mean $V$ magnitude suddenly began decreasing until 1977, fluctuating at intervals of $3.4 \pm 0.4$ yrs. with a variable, dampedlike amplitude whose maximum was nearly $0.4 \mathrm{mag}$. The intrinsic continuum reddening seems to be strongest when the star is brightest but not necessarily well correlated with line emission intensity as $\Phi_{\mathrm{rb}}$ remains high even in 1967 at a relative minimum of line emission. This reddening cleared up in 1977 , so that $\Phi_{\mathrm{rb}}$ became that of a B1V normal star for $D_{*}=0.116$ dex shown by a dashed vertical line in Fig. 6. Unfortunately, there are no photometric data corresponding to epochs of short-lived shell phases in 1973 and 1974. It is seen, however, that the $(U, D),(V, D),\left(\Phi_{\mathrm{rb}}, D\right)$ and $\left(\Phi_{\mathrm{rb}}, V\right)$ relations are single with $\partial(U, V) / \partial D>0$ and $\partial \Phi_{\mathrm{rb}} / \partial(D, V)<0$ slopes and that they are nicely conserved, even after the shell phases and the periods of loss of emission characteristics. A photometric microvariability of this star was observed, but there is no period determination at the present time.

\subsubsection{Stars most frequently seen in Be-shell phases}

\section{Pleione (HD 23862)}

Notable long-term changes are reported in the literature, for this star, see Hirata (1995) and references therein. Recently this star was discovered by Katahira et al. (1996) to be a binary with an orbital period of 218.0 days. We give here only a summary of spectral variations. Observed in a Be phase until 1904, Pleione entered a phase showing a rapidly rotating $\mathrm{B}$ normal-like star from 1905 to 1936. The first Be-metallic shell phase occurred in 1938 and ended in 1954. From 1954 to 1972, this star again entered a Be phase with strong Balmer and Fe II emission lines at maximum around 1960. Then gradual weakening began; in 1972, the $\mathrm{H} \alpha$ emission line was very weak. In late 1972 another metallic shell phase started and developed with a maximum near mid-1982. The metallic lines faded and disappeared in 1988 (Hirata 1995). The star entered a new Be phase; the $\mathrm{H} \alpha$ emission line gradually increased from 1982 (epoch of maximum strength in shell lines) to 1993 and decreased after. The global behaviour of spectral and light changes has been studied by many authors (Hirata \& Kogure 1976; Kogure \& Hirata 1982; Goraya \& Tur 1988). A steep decline in brightness occurred in 1938 and 1972 as each respective shell phase started. Furthermore, a strong weakening in the $U$ magnitude came with the maximum of metallic shell lines in 1982-1983.

From our study it is shown that in 1972, at the epoch of the appearance of a new Be-metallic shell phase associated with a rapid fainting in the $U B V$ colours, the $\Phi_{\mathrm{rb}}$ gradient rapidly increased and total $B D$ decreased indicating the contribution of a secondary $B D$ in emission. Between 1972 and 1988 the behaviour of the total $B D$ was the same as the strength of the metallic shell lines. It is interesting to note that the maximum value of the total $B D$ reported in 1982 corresponds to a maximum of the absorption strength of metallic lines. In the short interval of $D<D_{*}$, this star shows two $(U, D)$ and $(V, D)$ relations which are continued in the region of $D>D_{*}$, where normally in Be shell phases there is a single relation. The $\left(\Phi_{\mathrm{rb}}, D\right)$ and $\left(\Phi_{\mathrm{rb}}, V\right)$ seem to be single, unless for $D<D_{*}$ possible double relations are not well resolved. The $\left(\Phi_{\mathrm{rb}}, D\right)$ relation is slopeless, while $\left(\Phi_{\mathrm{rb}}, V\right)$ seems to have a global $\partial \Phi_{\mathrm{rb}} / \partial V>0$ slope. Rapid photometric variability was found in this object (McNamara 1987).

\section{Lib (HD 142983)}

$48 \mathrm{Lib}$ is one of the best examples of shell stars. The variable shell episode of $48 \mathrm{Lib}$ started in 1935: RV cycles $(8-13 \mathrm{yr})$, marked variations of intensity and in line profiles. These phenomena become more striking with increase of the amplitude of radial velocity (RV) (Aydin \& Faraggiana 1978 and references therein; Mon 1984; Hubert et al. 1987). In 48 Lib successive cycles exhibit negative RV phases longer than positive ones. The strength of shell lines has varied strongly since 1935. It was near or at maximum in 1943, 1965, 1970, 1974 and towards the end of 1993, according to recent observations published by Hanuschik et al. (1996). Maxima in the strength of emission in the first Balmer lines were generally seen at the same time or very close. Longterm photometric variations associated with spectroscopic cycles, and mid-term quasi-periodic oscillations (10-20 days) and sudden fadings (1-2 days) were reported by Mennickent et al. (1994) . These authors noted a brightness maximum in 1988 and possible minima in 1982 and 1991. In compiled data presented in this study, pronounced maxima of brightness were observed in 1976 and 1988. Balmer discontinuity is respectively lower and higher at epochs close to minima and maxima of the strength of shell metallic lines. Single relations are seen in $(U, D),(V$, $D),\left(\Phi_{\mathrm{rb}}, D\right)$ and $\left(\Phi_{\mathrm{rb}}, V\right)$. The $(U, D)$ has a $\partial U / \partial D>0$ slope, while in all others there is no slope. This star is a rapid photometric variable, which may in part explain the width of spectrophotometric relations.

\section{Her (HD 162732)}

88 Her is well-known to have presented associated longterm changes in its spectrum and in its light; in addition it was discovered by Harmanec et al. (1972) to be a binary ( $P=86.72$ days $)$. This star exhibited a Be phase with a hydrogen and metallic shell from 1955 to 1960. The $\mathrm{H} \alpha$ emission line gradually weakened from 1961 to 1971; the metallic shell lines, very weak from 1966, disappeared near 1970 (Atlas). In 1972-1976, the star was in a quasi-normal $\mathrm{B}$ phase, the $\mathrm{H} \alpha$ emission line was very weak in 1976-1977, and at the same epoch a minimum of the linear optical 
polarization was seen (Arsenijević et al. 1987). Then, the $\mathrm{H} \alpha$ emission line again gradually increased from 1977 to 1986. In addition, from 1978 to 1982 , a new metallic shell phase was increasing, followed from 1983 to 1986 by a decreasing metallic shell and mild Be phase (Doazan et al. 1982a; Hirata 1995). It was reported that the gradual weakening of Balmer emission and metallic shell lines in 1966-1970 was associated with brightening of the star (Doazan et al. 1982b) and that a large and rapid drop of the luminosity occurred near 1978, just before the development (1978-1982) of a new Be-shell metallic phase (Harmanec et al. 1980; Doazan et al. 1986; Hirata 1995). The polarization percentage rapidly increased in 1978, followed by a slow decline from 1979 to 1985 (Arsenijević et al. 1987).

From this study, it is seen that the total $B D$ was at minimum when the metallic shell lines disappeared towards 1970 and during the quasi-normal B phase (19721976). Then it gradually increased to mid-1982, in the same manner as the strength of shell lines. On the other hand, the star became brighter as the equivalent width of the $\mathrm{H} \alpha$ emission line increased (1978-1986), according to Hirata (1995). It is worth noting that $B C D$ observations of the stellar $B D$ from mid-1977 to the end of 1983 do not reveal any change in the photospheric $T_{\text {eff }}$ and $\log g$ parameters of this star during its phase changes (Zorec et al. 1989). The difference of brightness in the $U$ and $V$ magnitudes between the regions of $D<D_{*}$ and $D>D_{*}$ are clearly seen in Fig. 6. Single and slopeless relations $(U, D),(V, D),\left(\Phi_{\mathrm{rb}}, D\right)$ and $\left(\Phi_{\mathrm{rb}}, V\right)$ are characteristic for $D>D_{*}$. No rapid photometric variability was found in this object.

\subsection{Brief remarks on the remaining Be stars}

HD 28497. A rapid photometric variable star. The emission is strong when $D$ is small. The emission was low from 1989 to 1992 ; since then, $D$ and $V$ are increasing.

HD 35439. After a B normal-like phase, $\mathrm{H} \alpha$ emission appeared when $D$ was at its minimum value.

HD 37202. A shell and rapidly variable star. $D$ is at minimum when the strength of the shell is at minimum, but in such a case the RV is not at minimum in this star.

HD 37967. Emission is strong and there is almost no variation.

HD 48917. A rapidly variable star. Only a few spectroscopic data exist for this star.

HD 50123. Interacting binary having a composite B spectrum with a giant $\mathrm{K}$ companion. "Ellipsoidal" photometric variations with a period of $P=28.6$ days were found by Sterken et al. (1994).

HD 56014. Star with a moderate rotation $(V \sin i=$ $150 \mathrm{~km} \mathrm{~s}^{-1}$ ) showing at epochs a shell behaviour $D>D_{*}$.

HD 56139. A rapidly variable star with a small $V \sin i$ $\left(60 \mathrm{~km} \mathrm{~s}^{-1}\right)$ but sometimes showing the shell behaviour
$D>D_{*}$. The value of $I / I_{\mathrm{c}}$ for $\mathrm{H} \alpha$ is rather high $\left(I / I_{\mathrm{c}}=\right.$ $6)$. When $D$ is smallest, $\mathrm{H} \alpha$ emission is weak.

HD 58978. A helium-shell star. The strong spectroscopic variations in 1990 and in 1991 do not seem to be apparent for the spectrophotometric variations.

HD 60848. One of the hottest Be stars where the Balmer and Paschen line emission is well correlated with the Balmer continuum emission.

HD 60855. Star with moderate line emission showing $\mathrm{B} \leftrightarrows$ Be phase transitions. There are no strong spectrophotometric variations.

HD 63462. Star showing slight variations in the $\mathrm{H} \alpha$ emission intensity.

HD 65875. A rapidly variable star with moderate $V \sin i\left(150 \mathrm{~km} \mathrm{~s}^{-1}\right)$ and showing however phases with the shell behaviour $D>D_{*}$. The star has a high value of $I / I_{\mathrm{c}}$ in $\mathrm{H} \alpha\left(I / I_{\mathrm{c}}=9\right)$. Slopes $\partial(U, V) / \partial D<0$ and $\partial \Phi_{\mathrm{rb}} / \partial V>0$ seem to exist for this star.

HD 68980. Star with a very moderate $V \sin i$ $\left(95 \mathrm{~km} \mathrm{~s}^{-1}\right)$ and with very strong $\mathrm{H} \alpha$ emission $\left(I / I_{\mathrm{c}}=\right.$ 12). $D$ seems to be smallest when $\mathrm{H} \alpha$ emission is strongest. Two relations between $U, V$ versus $D$ and for $\Phi_{\text {rb }}$ against $V$ are apparent.

HD 83953. A rapidly variable star where $D$ is probably at maximum when the emission is low.

HD 89890. A star with a low rotation, $V \sin i=$ $70 \mathrm{~km} \mathrm{~s}^{-1}$, having $D>D_{*}$.

HD 91120. Emission is moderate with few variations.

HD 109387. A rapidly variable star. Emission is strong when $D$ is low and $\Phi_{\mathrm{rb}}$ is high. There is a high dispersion in the $(D, V)$ diagram for $D \sim D_{*}$ in 1986 .

HD 120991. Star with small $V \sin i\left(70 \mathrm{~km} \mathrm{~s}^{-1}\right)$. Sometimes $I / I_{\mathrm{c}}$ is high for $\mathrm{H} \alpha\left(I / I_{\mathrm{c}}=7\right.$ in 1993). $D$ diminishes when the emission increases. There are probably two correlations for $U$ and $V$ as a function of $D$.

HD 131492. A temporary strong shell phase was observed by Slettebak in 1980 which corresponded to a minimum in $V$ and to a maximum in $D$ (Slettebak 1982). This is responsible for the horizontal part in the diagram of $\Phi_{\mathrm{rb}}$ against $D$. The shell behaviour $D>D_{*}$ is clearly seen, although the star apparently has moderate rotation $\left(V \sin i=100 \mathrm{~km} \mathrm{~s}^{-1}\right)$.

HD 162428. The emission and the shell are variable.

HD 168797. This is a $\mathrm{B} \leftrightarrows \mathrm{Be}$ phase variable star. Emission is moderate.

HD 171780. A B $\leftrightarrows$ Be variable star with moderate emission.

HD 178175. Star observed in spectroscopy very irregularly. The shell behaviour $D>D_{*}$ is slightly present and the star has a moderate rotation $(V \sin i=$ $120 \mathrm{~km} \mathrm{~s}^{-1}$ ).

HD 183656. A rapidly variable shell star. $D$ is correlated with the $V / R$ variations (the $V / R$ and radial velocity curves are given in Koubsky et al. 1989). $D$ is at maximum when the RV is at maximum. 
HD 184279. A shell star, where $D$ is correlated with the magnitude $V$ and with the RV curve: $D$ is at maximum as RV is maximum (in 1979).

HD 187811. A B $\leftrightarrows \mathrm{Be}$ variable star with rather moderate emission.

HD 191610. A rapidly variable star. It has been seen in B phase in 1955 to 1958 , and in a Be phase since then. Emission is moderate.

HD 195325. A late Be star with a hydrogen and metallic shell.

HD 205637. A rapidly variable star where the hydrogen and metallic-line shell are variable.

HD 217050. A rapidly variable shell star. $D$ is at maximum when the strength of the shell is at maximum.

HD 217543. Star with moderate emission showing $\mathrm{B} \leftrightarrows$ Be phase transitions.

HD 218674. Star with rapid variability. It also shows strong emissions and a hydrogen-shell.

\subsection{Comments on the correlations obtained}

\subsubsection{General spectrophotometric behaviour}

We may conclude that patterns in Fig. 6 show that spectrophotometric changes in Be stars are characterized by $\left(U, V, \Phi_{\mathrm{rb}} ; D\right)$ and $\left(\Phi_{\mathrm{rb}}, V\right)$ relations which differ on the emission/absorption phase and may differ from star to star. More or less well-defined relations involving $D<D_{*}$ correspond to definite Be-phases. They can be either single or bivalued, mostly with $\partial(U, V) / \partial D>0$ and $\partial \Phi_{\mathrm{rb}} / \partial D<$ 0 , but sometimes also with slopes $\partial(U, V) / \partial D>0$ and $\partial \Phi_{\mathrm{rb}} / \partial D>0$. On the contrary, the most current shape of relation during $\mathrm{SPh}$-shell phases where $D>D_{*}$ can be summarized by: $\partial\left(V, \Phi_{\mathrm{rb}}\right) / \partial D \simeq 0$, and depending on the stars $\partial U / \partial D \simeq 0$ or $\partial U / \partial D>0$. The constancy of the magnitude $V$ is within 0.15 mag and sometimes even more. However, for some Be-shell stars, variation in $V$ can be as high as 0.25 mag.

In general, of those Be stars which have two correlations of $U, V$ against $D$, and, $\Phi_{\mathrm{rb}}$ against $V$ in Be phases $\left(D<D_{*}\right)$, nearly all have strong Balmer emission lines. The fact that there are two relations or only one is not related to particular values of $V \sin i$.

\subsubsection{Stars with low $V \sin i$ in $\mathrm{SPh}$-shell phases}

It is noteworthy the behaviour of some stars like HD 56014, HD 56139, HD 65875, HD 131492, where all have $D_{*}$ determined in the $B C D$ system, which show both $\mathrm{SPh}-\mathrm{Be}$ and $\mathrm{SPh}$-shell phases. The mean deviation of these objects for the SPh-shell phase is $\overline{D-D_{*}}=0.09 \pm 0.04$ dex which is more than 3 times the expected error $\epsilon(D)$ given in Table 3. Most of these deviations are depicted by data from the Geneva photometric system, which is one of the most stable and uniform (Sterken \& Manfroid 1991). These comments are also relevant to HD 178175 , where $D_{*}$ is only for the mean MK spectral type and the $D-D_{*} \leq 0.04$ dex deviations of the $\mathrm{SPh}$-shell like phase are established from the Geneva photometric data. In Fig. 6 we see that for most of the above mentioned stars, the transition between a $\mathrm{SPh}-\mathrm{Be}$ and a $\mathrm{SPh}$-shell phase is characterized by a change of slopes in the spectrophotometric correlations. It would be difficult to understand such slope changes in terms of errors affecting the BDs, as they would likely conserve one of the observed slopes.

Among those stars where both spectrophotometric behaviours: $\operatorname{Be}\left(\Delta V<0 ; D<D_{*}\right)$ and shell $(\Delta V \gtrsim 0$; $\left.D>D_{*}\right)$ were seen, there are some with small or moderate $V \sin i$. We also note that two Be stars with low $V \sin i$ were seen only in SPh-shell phases where $\Delta D>0$ $\left(\mathrm{HD} 89890\left(V \sin i=70 \mathrm{~km} \mathrm{~s}^{-1}\right)\right.$ and HD $178175(V \sin i=$ $120 \mathrm{~km} \mathrm{~s}^{-1}$ ). Low values of $V \sin i$ may correspond either to small $\sin i$ or to low velocity $V$. In the first case, the SPh-shell phase: $D>D_{*}$, cannot be explained by highly flattened CE seen pole-on. In the second case, we should admit the existence of an important fraction of slowly rotating Be stars (Mennickent et al. 1994). Nevertheless, for most Be-shell stars studied in this paper it is $\overline{V \sin i} \sim$ $300 \mathrm{~km} \mathrm{~s}^{-1}$.

On the other hand, it is also worth noting that in Beshell stars where enough RV data existed to be correlated with $D$ (HD 142983, HD 183656, HD 184279 etc.), the highest RV $>0$ appear when the line shell phenomenon and the absorption in the $B D$ due to the $\mathrm{CE}$ are strongest. This phenomenon might favor the formation of compact CE layers near the star.

\section{Discussion}

Using a simple phenomenological model for a star-CE system, we give in this section an outline for a discussion which aims at obtaining the first indications on the continuum opacity regimes of $\mathrm{CE}$ in Be stars, responsible for their most characteristic spectrophotometric changes, namely: (a) single relations in definite $\mathrm{SPh}-\mathrm{Be}$ phases with slopes $\partial V / \partial D>0$ and $\partial \Phi_{\mathrm{rb}} / \partial(D, V)<0$; (b) no variation of $V$ and $\Phi_{\mathrm{rb}}$ in $\mathrm{SPh}$-shell phases $\left(\partial\left(V, \Phi_{\mathrm{rb}}\right) / \partial D=0\right)$.

\subsection{Model of the CE}

Assuming an ellipsoidal $\mathrm{CE}$ with ellipticity $E=h / R_{\mathrm{e}}$ ( $h$ is the polar height of the $\mathrm{CE}$ and $R_{\mathrm{e}}$ its equatorial radius), the radiation flux emitted by a Be star can, in a first approximation, be described with an ellipsoidal slablike model as:

$F_{\lambda} / F_{\lambda}^{*} \simeq s_{\lambda}+\delta \times\left(1-\mathrm{e}^{-2 \tau_{\lambda}}\right)$

where the geometrical factor $\delta$, which is also proportional to the ratio of the envelope source function to the underlying stellar flux, is:

$\delta=\Omega \times\left[B_{\lambda}\left(T_{\text {env }}\right) / F_{\lambda}^{*}\right]$. 
In $(22)$ we have $s_{\lambda}=\mathrm{e}^{-\tau_{\lambda}}$ for $h>R_{*}$, where $\tau_{\lambda}=\tau_{\lambda}^{\circ}[1-$ $\left.\left(1-E^{2}\right) \sin i^{2}\right]^{-1 / 2}$ is the radial optical thickness of the shell. For cases where $h<R_{*}$ and $i \leq i_{\mathrm{h}}=\operatorname{arc} \cos \left(h / R_{*}\right)$ (polar regions of the star not shielded by the $\mathrm{CE}$ ) it is $s_{\lambda}=1$. In $(23) \Omega=\left(R_{\mathrm{e}} / R_{*}\right)^{2}\left[1-\left(1-E^{2}\right) \sin i^{2}\right]^{1 / 2}$ is the normalized projected area of the CE; $B_{\lambda}$ is the Planck function for the $\mathrm{CE}$ excitation temperature $T_{\text {env }} ; F_{\lambda}^{*}$ is the stellar flux. Except for $i \leq i_{\mathrm{h}}$ when $h<R_{*}$ so that $s_{\lambda}=1$, we see that $\tau_{\lambda}$ and $\delta$ are scaled by the same constant factor $\left[1-\left(1-E^{2}\right) \sin i^{2}\right]$. For a qualitative discussion of $(22)$ we may then use either a given value of $\left[1-\left(1-E^{2}\right) \sin i^{2}\right]$ or simply, for the sake of brevity, consider $E=1$, which corresponds to a spherical CE. This approximation was widely used in the literature to discuss visible energy distributions of Be stars. We also assume that the stellar components $\left(V_{*}, \Phi_{\mathrm{rb}}^{*}, D_{*}\right)$ are not variable (Zorec \& Briot 1991). Thus, using (22) with $E=1$, the magnitude excess $\Delta V=V-V_{*}$, the colour excess $\Delta \Phi_{\mathrm{rb}}=\Phi_{\mathrm{rb}}-\Phi_{\mathrm{rb}}^{*}$ and the $B D$ discrepancy $\Delta D=D-D_{*}$ produced by the $\mathrm{CE}$, can be represented as:

$$
\left.\begin{array}{ccc}
\Delta V\left(\delta, \tau_{\lambda_{\mathrm{V}}}\right) & = & -2.5 \times \log \left(F_{\lambda_{\mathrm{V}}} / F_{\lambda_{\mathrm{V}}}^{*}\right) \\
\Delta \Phi_{\mathrm{rb}}\left(\Phi_{\mathrm{e}}, \delta, \tau_{\lambda_{\mathrm{V}}}\right) & = & -\left[\mathrm{d} \ln \left(F_{\lambda} / F_{\lambda}^{*}\right) / \mathrm{d}(1 / \lambda)\right]_{\lambda_{\mathrm{V}}} \\
\Delta D\left(\Phi_{\mathrm{e}}, \delta, \tau_{\lambda_{\mathrm{V}}}\right) & = & \left.\log \left[F_{\lambda_{\mathrm{D}}^{+}} / F_{\lambda_{\mathrm{D}}^{+}}^{*}\right) /\left(F_{\lambda_{\mathrm{D}}^{-}} / F_{\lambda_{\mathrm{D}}^{-}}^{*}\right)\right]
\end{array}\right\}
$$

where $\lambda_{\mathrm{V}}=0.55 \mu \mathrm{m} ; \lambda_{\mathrm{D}}^{ \pm}$stand for the Paschen and Balmer sides of the continuum energy distribution at $\lambda_{\mathrm{D}}=$ $0.37 \mu \mathrm{m}$. The gradients are obtained using the classic definition (Allen 1973): $\Phi=5 \lambda-\mathrm{d} \ln F_{\lambda} / \mathrm{d}(1 / \lambda)$. The gradient $\Phi_{\mathrm{e}}$ is derived from $B_{\lambda_{\mathrm{V}}}\left(T_{\text {env }}\right)$, so that $\Phi_{\mathrm{e}} \propto 1 / T_{\text {env }}$.

Before going into details of (24), let us comment on the curves $\delta=\delta\left(\Delta V, \tau_{\lambda_{\mathrm{V}}}\right)$ shown in Fig. 7 which describe the main characteristics of $(22)$ regarding the photometric variations of Be stars. Depending on the sign of $\Delta V$, two behaviours can be distinguished: (a) that corresponding to $\Delta V<0$, which is typical for a $\mathrm{SPh}-\mathrm{Be}$ phase and where we always have $2 \delta>1$; (b) that of $\Delta V>0$, which corresponds to a SPh-shell phase and where it is always $0 \leq \delta \leq 1$. In (a) two distinct regimes may clearly be identified: $(i)$ a low opacity regime characterized by $\tau_{\lambda_{\mathrm{V}}}<\tau_{\text {or }} \sim 0.7|\Delta V|^{0.55}$ (vertical side of $\delta\left(\tau_{\lambda_{\mathrm{V}}}\right)$ curves); (ii) a high opacity regime, where $\tau_{\lambda_{\mathrm{V}}}>\tau_{\text {or }}$ (horizontal side of $\delta\left(\tau_{\lambda_{\mathrm{V}}}\right)$ curves $)$. Both regimes are schematically separated in Fig. 7 by dashed lines at $\tau_{\text {or }}$ (to sketch out the separation of opacity regimes $\tau_{\text {or }}$ was choosen so that $\left.\partial \delta / \partial \tau_{\mathrm{V}}=-1\right)$. In Fig. 7, it can also be seen that in the low opacity regime: $\tau_{\lambda_{\mathrm{V}}}<\tau_{\text {or }}$, changes in the magnitude $V$ are mainly an opacity effect, because even if $\delta$ do not remain constant, small variations of $\tau_{\lambda_{\mathrm{V}}}$ will introduce marked changes of $\Delta V$. On the contrary, when $\tau_{\lambda_{\mathrm{V}}}>\tau_{\text {or }}$, variations in $V$ mostly reflect changes of $\delta$. In (b), that is, for a SPh-shell behaviour, $\tau_{\lambda_{\mathrm{V}}} \geq \tau_{\mathrm{sh}}=0.92 \Delta V$, which corresponds to a high opacity regime. Curves $\delta(\Delta \Phi)$ and $\delta(\Delta D)$ against $\tau_{\lambda_{\mathrm{V}}}$ also have the same kind of patterns as $\delta(\Delta V)$, but their dependences on $\Phi_{\mathrm{e}}$ and $\Delta D$ reveal some more subtle behaviours.
We note that the sign of $\Delta V$ is a function of $\delta$ and $\tau_{\lambda_{\mathrm{V}}}$ only. Hence, the magnitude changes corresponding to both spectrophotometric phase variations in the same star can be described with a simple spherical CE. In the next subsection we shall also see that for the same star this model is enough to explain the main spectrophotometric characteristics of both $\mathrm{SPh}-\mathrm{Be}$ and $\mathrm{SPh}-$ shell phases. We note again that for flattened $\mathrm{CE}$ with $h \leq R_{*}$ seen at angles $i \lesssim i_{\mathrm{h}}$, as $s_{\lambda}=1$ we cannot have $\Delta V>0$. On the other hand, it may happen that $\Delta V=0$, even if $\delta \neq 0$ and $\tau_{\lambda_{\mathrm{V}}} \neq 0$.

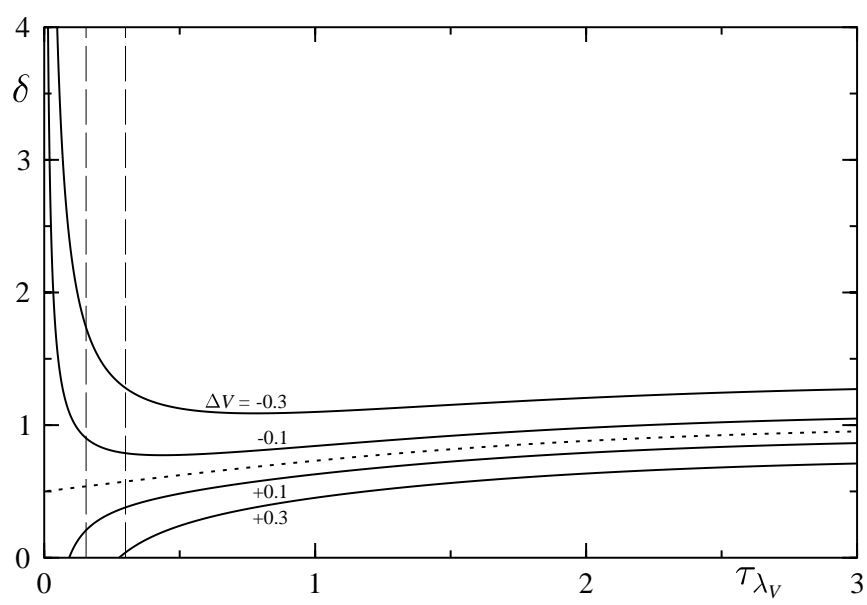

Fig. 7. Ratio $\delta$ (for $E=1$ ) as a function of optical depth $\tau_{\lambda_{\mathrm{V}}}$ for several values of $\Delta V$ corresponding to Be phases $(\Delta V<0)$ and Be-shell phases $(\Delta V>0)$. The vertical dashed lines schematically divide zones of low and high opacity for the respective $\Delta V<0$. The dotted curve corresponds to $\Delta V=0$

\subsection{Spectrophotometric variations}

In patterns of Fig. 6, two spectrophotometric behaviours seem to be the most relevant: (a) in SPh-Be phases, where $\Delta D=D-D_{*}<0$, there are linear correlations between $(V, D)$ and $\left(\Phi_{\mathrm{rb}}, D\right)$ so that $\partial V / \partial D>0$ and $\partial \Phi_{\mathrm{rb}} / \partial(D, V)<0$; (b) in shell phases, where $\Delta D>0$, there is a very small variation, if any, of $\Phi_{\mathrm{rb}}$ and $V$ as a function of $D$. Let us then see in what opacity regime these variations can take place.

\subsubsection{Low opacity regime}

For $\tau_{\lambda_{\mathrm{V}}}<\tau_{\text {or }}<1$, where $\delta>1$, from (24) we derive the following relations:

$$
\left.\begin{array}{ccc}
\Delta V & \sim & -0.9 \tau_{\lambda_{\mathrm{V}}}(2 \delta-1) \\
\Delta \Phi_{\mathrm{rb}} & \sim \tau_{\lambda_{\mathrm{V}}}\left[\delta\left(2 \Delta \Phi_{\mathrm{e}}+6 \lambda_{\mathrm{V}}\right)-3 \lambda_{\mathrm{V}}\right] /\left(1+2 \tau_{\lambda_{\mathrm{V}}} \delta\right)
\end{array}\right\}
$$


where $a=\tau_{\lambda_{\mathrm{D}}^{-}} / \tau_{\lambda_{\mathrm{D}}^{+}} \sim 3.4 \exp \left(2.2 \times 10^{4} / T_{\text {env }}\right) \gg 1$, with $\tau_{\lambda_{\mathrm{D}}}^{+} \simeq\left(\lambda_{\mathrm{D}} / \lambda_{\mathrm{V}}\right)^{3} \tau_{\lambda_{\mathrm{V}}}$. Using (25) we readily realize that:

$\Delta V / \Delta D>0 ; \Delta \Phi_{\mathrm{rb}} / \Delta D<0 ; \Delta \Phi_{\mathrm{rb}} / \Delta V<0$

which are characteristic for SPh-Be phases. This suggests that $\mathrm{SPh}-\mathrm{Be}$ variations are likely to be produced by $\mathrm{CE}$ in low opacity regimes. As for $\tau_{\lambda_{\mathrm{V}}}<1$ it is $T_{\text {env }} \simeq$ const., changes of $\delta$ as a function of $\tau_{\lambda_{\mathrm{V}}}<1$ represent variations of the CE extent $R_{\mathrm{e}} / R_{*}$. It follows from (22) that for a given value of $\Delta V \lesssim 0$ when $\tau_{\lambda_{\mathrm{V}}}<1, \delta$ (and so $R_{\mathrm{e}} / R_{*}$ ) is an increasing function of $1 / \tau_{\lambda_{\mathrm{V}}}$ that easily reach $2 \delta \geq 1$, which implies a rather extended CE. To an order of magnitude estimate, if we assume a B2e star $\left(T_{\text {eff }} \simeq 22500 \mathrm{~K}\right), \Delta V \simeq-0.2 \mathrm{mag}$ and $\tau_{\lambda_{\mathrm{V}}} \simeq 0.05$ for $T_{\text {env }} \sim 10^{4} \mathrm{~K}$, it follows from $(22)$ that $R_{\mathrm{e}} / R_{*} \simeq 3.5$.

\subsubsection{High opacity regime}

Two situations can be distinguished: (a) $\Delta V<0$, which implies emission excess in the Paschen continuum; (b) $\Delta V>0$, which indicates flux deficiency.

In (a) we have $\tau_{\lambda_{\mathrm{V}}}>\tau_{\text {or }}$ and:

$0.5 \mathrm{e}^{\tau_{\text {or }}} \lesssim \delta\left(\tau_{\lambda_{\mathrm{V}}}\right)<10^{-0.4 \Delta V}$

where mostly $\Delta D<0$.

In (b) it is $\tau_{\lambda_{\mathrm{V}}}>\tau_{\mathrm{sh}}$ and it always happens that:

$\delta\left(\tau_{\lambda_{\mathrm{V}}}\right)<1$

but the behaviour of $\Delta D$ deserves some additional explanations. Following the comments given in Sect. 3, the typical spectrophotometric behaviour in $\mathrm{SPh}$-shell phases seems to be summarized by:

$$
\left.\begin{array}{cc}
\partial \Delta \Phi_{\mathrm{rb}} / \partial D & =0 \\
\partial \Delta V / \partial D & =0
\end{array}\right\}
$$

Using (29) in (24), we derive a differential equation for $\Delta \Phi_{\mathrm{e}}=\Phi_{\mathrm{e}}-\Phi_{\mathrm{rb}}^{*}$ whose solution for $\tau_{\mathrm{sh}}<\tau_{\lambda_{\mathrm{V}}} \lesssim 1$ and for a wide range of $\Delta V>0$ can roughly be represented by:

$\Delta \Phi_{\mathrm{e}} \simeq 0.3 \tau_{\lambda_{\mathrm{V}}}^{0.9}$

Hence, knowing that:

$\Phi_{\mathrm{e}} \propto 1 / T_{\mathrm{env}}$

conditions (29) imply that $\mathrm{SPh}$-shell phases reveal sensitivity of $\mathrm{CE}$ to temperature changes of the $\mathrm{CE}$ and that $T_{\text {env }}$ is a decreasing function of $\tau_{\lambda_{\mathrm{V}}}$. On the other hand, with (24) and (30), it can be shown numerically that the $B D$ difference $\Delta D$ increases with $\tau_{\lambda_{\mathrm{V}}}$. So, the increase of $D$ in SPh-shell phases is probably due to $\mathrm{CE}$ in high opacity regimes with a decreasing temperature as the opacity becomes higher.

As noted before for $\Delta V$, it is also not possible to produce $F_{\lambda}<F_{\lambda}^{*}$ at $\lambda<\lambda_{\mathrm{D}}$ to have $\Delta D>0$, as needed for
SPh-shell phases in pole-on Be stars with flattened CE where $h \leq R_{*}$.

Relation (28) implies that in SPh-shell phases CE are rather shrunk. As in the preceding section, we assume that for a shell phase of a B2e star $\Delta V \simeq 0.2 \mathrm{mag}$ and $\tau_{\lambda_{\mathrm{V}}} \simeq 1$ are characteristic parameters. Writing $T_{\text {env }}=\epsilon T_{\text {eff }}$ where for most shell stars $\epsilon \simeq 0.5$ (Zorec \& Garcia 1991) we get $T_{\text {env }} \lesssim 14700 \mathrm{~K}$ so that $R_{\mathrm{e}} / R_{*} \simeq 1.3$. Using spectroscopic data, Kogure (1989) also concluded that regions of the CE responsible for spectroscopic shell spectra are smaller and denser than those producing spectroscopic Be phases. A more detailed discussion of these phenomena will be presented in a following paper.

\section{Conclusions}

In this work we studied the long-term spectrophotometric variations of 49 classic Be stars. The parameters used in this study are: the $U$ and $V$ magnitudes of the $U B V$ photometric system, total Balmer discontinuity $D$, and the gradient of the energy distribution $\Phi_{\mathrm{rb}}$ defined for $\lambda \lambda 0.40$ to $0.63 \mu \mathrm{m}$. To obtain these quantities for as long a time base as possible, we used genuine $B C D$ spectrophotometric data and photometric data obtained in most cases since 1950 in five different photometric systems: $U B V$, $U B V R I$, Geneva system, Strömgren's uvby and 13-colour system, which we reduced to a common spectrophotometric scale given by the $B C D$ system.

Variations of the $\left(V, D, \Phi_{\mathrm{rb}}\right)$ parameters were qualitatively compared with the spectroscopic variation of a small number of well-studied Be stars, which were mostly observed either in Be phases or in shell phases. From this comparison it generally follows that line emission is stronger when stars are brighter and redder, and that for strong reddening a veiling in the lines is also observed. Total Balmer discontinuity is smallest at phases of strong emission. In Be-shell phases the strength of the shell is always stronger when total Balmer discontinuity is greatest. RV are also highest in these cases.

For SPh-Be phases total Balmer discontinuity is smaller than the stellar component and for each star there are well-defined $(U, D),(V, D),\left(\Phi_{\mathrm{rb}}, D\right)$ and $\left(\Phi_{\mathrm{rb}}, V\right)$ linear-like correlations which can be single or doublevalued. Slopes of these correlations are $\partial(U, V) / \partial D>0$ and $\partial \Phi_{\mathrm{rb}} / \partial(D, V)<0$ in most cases, but sometimes their signes are changed. In SPh-shell phases the total $B D$ is higher than the stellar $B D$, and generally no variation of $V$ and $\Phi_{\mathrm{rb}}$ accompanies the increase of $D$. In some rare cases a slight variation of $V$ and $\Phi_{\mathrm{rb}}$ and even a bluening is observed near $D \gtrsim D_{*}$. However, when a spectroscopic shell spectrum develops, reddenings or bluenings may sometimes be spurious effects due to shell lines entering the photometric filter pass-bands which are not considered in the calibration of photometric indices in spectrophotometric quantities. 
The fact that the spectrophotometric patterns are single or double-valued does not seem to correlate with $V \sin i$.

For a number of Be stars a unique pattern was observed for both ranges of $D, D \gtrless D_{*}$, even after having passed through several shell phases and apparent loss of emission characteristics. Slopes of these patterns are different whether it is $D<D_{*}$ or $D>D_{*}$.

SPh-shell behaviours such that $D>D_{*}$ have been observed for several stars which have moderate $V \sin i$ parameters. If these values of $V \sin i$ are due to small aspect angles $i$, this will mean that in such stars the CE in the neighbouring stellar regions cannot be strongly flattened.

Sometimes, the spectrophotometric correlations are somewhat dispersed. This phenomenon may possibly be due to high photometric variability, produced by activities in the underlying stellar surface, which does not obey the "smoothed" long-term variation due to the CE.

We showed that the spectrophotometric variations in SPh-Be phases may well be produced by $\mathrm{CE}$ in low opacity regimes $\left(\tau_{\lambda_{\mathrm{V}}}<\tau_{\mathrm{or}}, \Delta V<0\right)$. Those observed in $\mathrm{SPh}-$ shell phases are due to $\mathrm{CE}$ in high opacity regimes $\left(\tau_{\lambda_{\mathrm{V}}}>\right.$ $\tau_{\mathrm{sh}}, \Delta V>0$ ), where the temperature decreases as their opacity increases. The low opacity $\mathrm{CE}$ seem to be more extended than those of high opacity. The high positive values of RV observed in the stronger shell phases should probably favor the appearance of compact formations near the star.

The formation of CE in Be stars, as well as their structure, are still open questions. They may not only depend on the mass loss mechanisms, but also on the way the mass is lost and its dynamics. Polarimetric data cannot be interpreted if $\mathrm{CE}$ are not somewhat flattened. Nevertheless, recent interferometric measurements of some Be stars (Stee et al. 1995, 1997; Quirrenbach et al. 1997) reveal that the effective ellipticities of $\mathrm{CE}$ for the $\mathrm{H} \alpha$ line formation region are $E \sim 0.4 \pm 0.3$, which clearly rule out the spherical geometry of CE regions exceeding $R_{\mathrm{e}} \sim 6 R_{*}$. However, for the visible continuum, for which $R_{\mathrm{e}} \lesssim 4 R_{*}$ (Stee et al. 1997), such an approximation can still be reliable. The present study allowed us to appreciate some systematic spectrophotometric behaviours of Be stars. In particular, it showed that not only $\mathrm{SPh}$-Be and $\mathrm{SPh}$-shell phases can be present in a same star, but that they can both take place in stars with low values of $V \sin i$. If these low $V \sin i$ are due to $\sin i, \mathrm{CE}$ geometries preventing the shielding of stellar polar regions: high flattened disks, lemniscate shaped $\mathrm{CE}$ at $R_{\mathrm{e}} \lesssim 4 R_{*}$, should probably be not appropriate to model the spectrophotometric behaviour of these objects. In order to better constrain models of $\mathrm{CE}$ for Be stars (their geometries in particular) and/or understand the phenomena underlying the observed spectrophotometric behaviours, a number of complementary observational and/or quantitative studies have still to be done. Correlations of spectrophotometric vs. spectroscopic behaviours, time lags between both, as well as a study of the properties of spectrophotometric relations presented in this paper against the stellar fundamental parameters and the aspect angle $i$ will be presented in a next contribution. Using different model envelopes, a quantitative discussion in terms of opacity, size and temperature of $\mathrm{CE}$ will complete the present work.

Acknowledgements. Many thanks are due to Dr. Ch. Sterken for having helped us to manage the data archives of the LongTerm Photometry of Variables (LTPV) program at ESO. We are indebted to Dr. J. Percy, who kindly provided us with some unpublished photometric data and to all those observers whose work is included in the IAU Archive of Unpublished Photoelectric Observations (accessible by CDS). We are particularly grateful to Dr. P. Harmanec and Dr. K. Pavlovski for having provided us before publication with some $U B V$ data collected at the Hvar Observatory. Discussions with Dr. S. Štefl on 59 Cyg are warmly acknowledged. Useful comments and painstaking reading of the manuscript by the referee, Pr. J. Dachs, which helped to improve the presentation of the paper are greatly acknowledged. This research has made use of several databases and abstract services: Centre de Données Stellaires de Strasbourg (CDS), The General Catalogue of Photometric Data (GCPD, University of Lausanne), Astronomical Data Analysis Center (ADAC, NAO Japan) and NASA's Astrophysics Data System Abstract Service (ADS).

\section{References}

Adelman S.J., 1992, PASP 104, 392

Allen C.W., 1973, Astrophysical Quantities, University of London

Alvarez M., Schuster W.J., 1981, Rev. Mex. Astron. Astrofis. 6,163

Alvarez M., Schuster W.J., 1982, Rev. Mex. Astron. Astrofis. 5,173

Andrillat Y., Fechrenbach C., 1982, A\&AS 48, 93

Apparao K.M.V., 1991, ApJ 376, 256

Appenzeller I., 1966, Z. Astrophys. 64, 269

Ardeberg A., Wramdemark S., 1970, Ark. Astr. 5, 387

Arsenijević J., Jankov S., Djurašević G., 1987, Long-term Polarization Changes of 88 Her. In: Slettebak A., Snow T. (eds.), Physics of Be Stars, IAU Coll. No. 92. Cambridge Univ. Press, p. 101

Aydin C., Faraggiana R., 1978, A\&AS 34, 51

Baade D., Balona L.A., 1994, in: Balona L.A., Henrichs H.F., Le Contel J.M. (eds.), Pulsation, Rotation and Mass Loss in Early-Type Stars, IAU Symp. No. 162. Kluwer Acad. Publ., p. 311

Baldinelli L., Ferri A., Ghedini S., 1981, Inf. Bull. Var. Stars 1993

Ballereau D., Chauville J., 1987, Rev. Mex. Astron. Astrofis. 15,29

Ballereau D., Chauville J., Zorec J., 1995, A\&AS 111, 423

Balona L.A., Cuypers J., Marang F., 1992, A\&AS 92, 533

Balona L.A., 1990, MNRAS 245, 92

Balona L.A., 1995, MNRAS 277, 1547

Barbier D., 1947, Ann. Astrophys. 10, 13

Barbier D., Chalonge D., 1939, ApJ 90, 627

Barbier D., Chalonge D., 1941, Ann. Astrophys. 4, 30 
Barrera L.H., Mennickent R.E., Vogt N., 1991, Ap\&SS 185, 79

Barylak M., Doazan V., 1986, A\&A 159, 65

Belyakina T.S., Chugainov P.F., 1960, Izv. Krymskoj Astr. Obs. 22, 257

Bernacca P.L., Bianchi L., 1981, A\&A 94, 345

Böhme D., 1984, Inf. Bull. Var. Stars 2507

Böhme D., 1985, Inf. Bull. Var. Stars 2723

Böhme D., 1986, Inf. Bull. Var. Stars 2893

Böhme D., 1988, Inf. Bull. Var. Stars 3222

Bouigue R., 1959, Ann. Obs. Toulouse 27

Božič H., Harmanec P., Horn J., et al., 1995, A\&A 304, 235

Brodskaja E.S., 1968, Per. Zvezd. 16, 429

Brucato R.J., Kristian J., 1972, ApJ 173, L105

Buscombe W., 1977, MK Spectral Classification, Third General Catalogue, Evanston

Buscombe W., 1980, MK Spectral Classification, Fourth General Catalogue, Evanston

Buscombe W., 1981, MK Spectral Classification, Fifth General Catalogue, Evanston

Buscombe W., 1988, MK Spectral Classification, Seventh General Catalogue, Evanston

Cameron R.C., 1966, Georgetown Obs. Monog. No. 21

Catalano S., Umana G., 1987, Photometric and $\mathrm{H} \alpha$ Variability of Some Be Stars, in: Slettebak A., Snow T. (eds.), Physics of Be Stars. IAU Coll. No. 92. Cambridge Univ. Press, p. 101

CETAMA, 1978, Statistique Appliquée à l'Exploitation des Mesures, Commissariat à l'Énergie Atomique, Masson

Chalonge D., Divan L., 1952, Ann. Astrophys. 15, 201

Chalonge D., Divan L., 1973, A\&A 23, 69

Chalonge D., Safir H., 1936, C.R. Acad. Sci. Paris 203, 1329

Chkhikvadze Y.N., 1980, Astrofizika 16, 411

Chochol D., Bakos G.A., Bartolini C., 1985, Contr. Astron. Obs. Skalnate Pleso 13, 75

Clariá J.J., Escosteguy L.A., 1981, PASP 93, 636

Corben P.M., 1966, Mon. Not. Astr. Soc. S. Africa 25, 44

Corben P.M., 1971, Mon. Not. Astr. Soc. S. Africa 30, 37

Cousins A.W.J., 1962, Mon. Not. Astron. Soc. S. Africa 21, 20

Cousins A.W.J., Stoy R.H., 1963, Royal Obs. Bull. 64, 1

Cousins A.W.J., 1964, Mon. Not. Astron. Soc. S. Africa 23, 175

Cousins A.W.J., 1965, Mon. Not. Astron. Soc. S. Africa 24, 120

Cousins A.W.J., 1973, Mon. Not. Astron. Soc. S. Africa 32, 11

Cousins A.W.J., 1976, Mem. R. Astron. Soc. 81, 25

Cousins A.W.J., 1978, Mon. Not. Astron. Soc. S. Africa 37, 8

Cousins A.W.J., Stoy R.H., 1962, Royal Obs. Bull. 64

Cousins A.W.J., Stoy R.H., 1970, Mon. Not. Astr. Soc. S. Africa 29, 91

Cowley A.P., McLaughlin D.B., Toney J., et al., 1972, PASP 84,834

Crawford D.L., 1963a, ApJ 137, 523

Crawford D.L., 1963b, ApJ 137, 530

Crawford D.L., Barnes J.V., 1970, AJ 75, 978

Crawford D.L., Barnes J.V., Faure B.Q., et al., 1966, AJ 71, 709

Crawford D.L., Barnes J.V., Golson J.C., 1970, AJ 75, 624

Crawford D.L., Barnes J.V., Golson J.C., 1971a, AJ 76, 621

Crawford D.L., Barnes J.V., Golson J.C., 1971b, AJ 76, 1058

Crawford D.L., Barnes J.V., Golson J.C., 1973, AJ 78, 738
Cruzado A., Cidale L., Zorec J., 1994, Structure of the Envelope of 48 Lib. In: Balona L.A., Henrichs H.F., Le Contel J.M. (eds.), Pulsation, Rotation and Mass Loss in Early-Type Stars, IAU Symp. No. 162. Kluwer Acad. Publ., p. 366

Dachs J., 1982, A Study of Be Star Variability. In: Jaschek M., Groth H.G. (eds.) IAU Symp. No. 98, Be Stars. Reidel Publ. Co., p. 19

Dachs J., Engels D., Kiehling R., 1988, A\&A 194, 189

Dachs J., Hanuschik R., 1984, A\&A 138, 140

Dachs J., Hanuschik R., Kaiser D., et al., 1986, A\&AS 63, 87

Dachs J., Poetzel R., Kaiser D., 1989, A\&AS 78, 487

Dahn C.C., Gueter H.H., 1973, ApJ 179, 551

Danks A.C., Houziaux L., 1978, PASP 90, 453

Dapergolas A., Di Cola G., Guarnieri A., et al., 1981, Inf. Bull. Var. Stars 1920

de Loore C., Altamore A., Baratta G.B., et al., 1979, A\&A 78, 287

Denoyelle J., 1977, A\&AS 27, 343

Deutschman W.A., Davis R.J., Schild R.E., 1976, ApJS 30, 97

Divan L., 1966, Spectrophotometric Standards in Use at Paris. In: Lodén K., Lodén L.O., Sinnerstad U. (eds.), Spectral Classification and Multicolour Photometry, IAU Symp. No. 24, p. 311

Divan L., 1979, Spectral classification of Be stars. In: McCarthy M.F., Philip A.G.D., Coyne G.V. (eds.), IAU Coll. No. 47, Spectral Classification of the Future. Vatican Obs., p. 247

Divan L., Zorec J., 1982a, Basic parameters of O and B stars. In: Perrymann M.A.C., Guyenne T.D. (eds.), The Scientific Aspects of the Hipparcos Mission. ESA-SP 177, p. 101

Divan L., Zorec J., 1982b, Energy distribution of 59 Cyg. In: Third European IUE Conference. ESA-SP 176, p. 291

Divan L., Zorec J., 1982c, BCD spectrophotometry of the Beshell star 88 Her. In: Jaschek M., Groth H.G. (eds.) IAU Symp. No. 98, Be Stars. Reidel Publ. Co., p. 61

Divan L., Zorec J., Andrillat Y., 1983, A\&A 126, L8

Divan L., Zorec J., Briot D., 1978, The Messenger (ESO) No. 14. 11

Divan L., Zorec J., Briot D., 1982, Correlation between $B C D$ parameters of Be stars. In: Jaschek M., Groth H.G. (eds.), IAU Symp. No. 98. Be Stars, Reidel Publ. Co., p. 53

Doazan V., Barylak M., Rusconi L., et al., 1989, A\&A 210, 249

Doazan V., Franco M., Rusconi L., et al., 1983, A\&A 128, 171

Doazan V., Harmanec P., Koubsky P., et al., 1982a, A\&AS 50, 481

Doazan V., Harmanec P., Koubsky P., et al., 1982b, A\&A 115, 138

Doazan V., Rusconi L., Sedmak G., et al., 1987, A\&A 182, L25

Doazan V., Thomas R.N., Barylak M., 1986, A\&A 159, 75

Echevarría J., Roth M., Warman J., 1979, Rev. Mex. Astron. Astrofis. 4, 287

Eggen O.J., 1955, AJ 60, 65

Eggen O.J., 1963a, AJ 68, 483

Eggen O.J., 1963b, AJ 68, 697

Eggen O.J., 1968, Royal Obs. Bull. No. 137

Elliot J.E., 1974, AJ 79, 1082

Erro B.I., 1969, Bol. Inst. Tonantzintla Tacubaya 5, 89

Feinstein A., 1967, ApJ 149, 107

Feinstein A., 1968a, Z. Astrophys. 68, 29

Feinstein A., 1968b (priv. comm. in The General Catalogue of Photometric Data, Mermilliod J.C., Hauck B., 
Mermilliod M., Univ. of Lausanne, Switzerland: http://obswww.unige.ch/gcpd/gcpd.html)

Feinstein A., 1970, PASP 82, 132

Feinstein A., 1974, MNRAS 169, 171

Feinstein A., 1975, PASP 87, 603

Feinstein A., 1980 (priv. comm. in A\&A 138, 140)

Feinstein A., Marraco H., 1979, AJ 84, 1713

Fernie J.D., 1991, Inf. Bull. Var. Stars 3558

Ferrari-Toniolo M., Natali G., Persi P., et al., 1977, A\&A 61, 47

Ferrari-Toniolo M., Persi P., Viotti R., 1978, MNRAS 185, 841 Franco G.A.P., 1989, A\&AS 80, 127

Frohlich A., Nevo I., 1974, 167 MNRAS 167, 221

Garrison R.F., Kormendy J., 1976, PASP 88, 865

Gerasimovič B.P., 1929, Harvard Circ. 339

Gebbie K.B., Thomas R.N., 1970, ApJ 161, 229

Gebbie K.B., Thomas R.N., 1971, ApJ 168, 461

Golay M., 1958, Publ. Obs. Genève, Ser. A 57, 374

Golay M., 1963, Publ. Obs. Genève, Ser. A 64, 199

Golay M., 1973, Remarks on the Photometric Criteria of Choice of the Standard Stars. In: Hauck B., Westerlund B.E. (eds.), Problems of Calibration of Absolute Magnitudes and Temperature of Stars, IAU Symp. No. 54. Reidel Pub. Co., Dordrecht, p. 275

Goraya P.S., Tur N.S., 1988, A\&A 205, 164

Gray R.O., Olsen E.H., 1991, A\&AS 87, 541

Greaves W.M.H., Martin E., 1938, MNRAS 98, 434

Grønbech B., Olsen E.H., 1976, A\&AS 25, 213

Grønbech B., Olsen E.H., Strömgren B., 1976, A\&AS 26, 115

Grønbech B., Olsen E.H., 1977, A\&AS 27, 433

Guetter H.H., 1974, PASP 86, 795

Gulliver A.F., 1983 (priv. comm. in A\&AS 107, 403)

Guo Y., Huang L., Hao J., et al., 1995, A\&AS 112, 201

Gutierrez-Moreno A., 1975, PASP 87, 805

Gutierrez-Moreno A., Moreno H., 1968, ApJS 15, 459

Gutierrez-Moreno A., Moreno H., Stock J., et al., 1966, Publ. Univ. Chile No. 1

Häggkvist L., 1971, A\&A 12, 5

Häggkvist L., Oja T., 1966, Ark. Astr. 4, 137

Häggkvist L., Oja T., 1969, Ark. Astr. 5, 303

Häggkvist L., Oja T., 1970 (priv. comm. in The General Catalogue of Photometric Data, Mermilliod J.C., Hauck B., Mermilliod M., Univ. of Lausanne, Switzerland: http://obswww.unige.ch/gcpd/gcpd.html)

Hanuschik R., Hummel W., Sutorius E., et al., 1996, A\&AS 116,309

Hardie R.H., Crawford D.L., 1961, ApJ 133, 843

Harmanec P., Horn J., Juza K., 1994, A\&AS 104, 121

Harmanec P., Horn J., Koubsky P., et al., 1978, Bull. Astron. Inst. Czech. 29, 278

Harmanec P., Horn J., Koubsky P., et al., 1980, Bull. Astron. Inst. Czech. 31, 144

Harmanec P., Koubsky P., Krpata J., 1972, Bull. Astron. Inst. Czech. 23, 218

Harmanec P., Kř́íz S., 1976, in: Slettebak A. (ed.), Be and Shell Stars, IAU Symp. No. 70. Reidel, p. 385

Harris III D.L., 1955, ApJ 121, 554

Harris III D.L., 1956, ApJ 123, 371

Hauck B., 1985, Calibration in Temperature of Photometric Parameters. In: Hayes D.S., Pasinetti L.E., Philip A.G.D. (eds.), Calibration of Fundamental Stellar Quantities, IAU Symp. No. 111. Dordrecht, p. 271

Hauck B., Mermilliod M., 1975, A\&AS 22, 235

Haug U., 1970, A\&A 9, 453

Haupt H., 1974, Inf. Bull. Var. Stars 928

Haupt H.F., Moffat A.F.J., 1973, ApJL 13, 77

Haupt H.F., Schroll A., 1974, A\&AS 15, 311

Heck A., Manfroid J., 1980, A\&AS 42, 311

Hill P.W., 1970, MNRAS 150, 23

Hiltner W.A., 1956, ApJS 2, 389

Hiltner W.A., Johnson H.L., 1956, ApJ 124, 367

Hirata R., 1982, Long-term variation of Be stars on the colormagnitude diagram. In: Jaschek M., Groth H.G. (eds.) IAU Symp. No. 98. Be Stars. Reidel Publ. Co., p. 41

Hirata R., Hubert-Delplace A.M., 1981, Variability of Be stars. In: G.E.V.O.N. and Sterken C. (eds.), Workshop on pulsating B stars, Obs. de Nice, p. 217

Hirata R., 1984, Interpretation of photometric activity in Be stars. In: Pecker J.C., Uchida Y. (eds.), Japan-France Seminar, Active Phenomena in the Outer Atmosphere of the Sun and Stars, CNRS and Obs. de Paris, p. 115

Hirata R., 1995, PASJ 47, 195

Hirata R., Kogure T., 1976, PASJ 28, 509

Hoag A.A., Johnson H.L., Iriarte B., et al., 1961, Pub. US. Nav. Obs. 17, Part VII, 347

Hoffleit D., Jaschek C., 1982, The Bright Star Catalogue, Yale Univ. Obs.

Hoffleit D., Saladyga M., Wlasuk P., 1983, A Supplement to the Bright Star Catalogue, Yale Univ. Obs.

Hogg A.R., 1958, Mount Stromlo Obs. Mimeo. No. 2

Hopp U., Witzigmann S., 1980, Inf. Bull. Var. Stars 1782

Hopp U., Witzigmann S., Geyer E.H., 1982, Inf. Bull. Var. Stars 2148

Horaguchi T., Kogure T., Hirata R., et al., 1994, PASJ 46, 9

Horn J., Kubat J., Harmanec P., et al., 1996, A\&AS 309, 521

Horn J., Božič H., Harmanec P., et al., 1982, Bull. Astron. Inst. Czech. 33, 308

Howarth I.D., 1979, J. Brit. Astron. Assoc. 89, 378

Hubert-Delplace A.M., Hubert H., 1979, An Atlas of Be Stars. Obs. de Paris-Meudon and Obs. de Haute-Provence

Hubert-Delplace A.M., Hubert H., 1981, A\&AS 44, 109

Hubert-Delplace A.M., Hubert H., Ballereau D., et al., 1982, Recent changes of the Be star HD 58050. In: Jaschek M., Groth H.G. (eds.) IAU Symp. No. 98. Be Stars. Reidel Publ. Co., p. 195

Hubert-Delplace A.M., Floquet M., Chambon M.T., 1987, A\&A 186, 213

Hutchings J.B., 1977, MNRAS 181, 619

Hutchison R., 1974 (priv. comm. in PASP 86, 894)

Huffer C.M., 1939, ApJ 89, 139

Iliev L., Kovachev B., Tomov N., 1991, Astrophys. Inv. (Bulgarian Acad. Sci.) 6, 47

Iriarte B., 1965, Bol. Inst. Tonantzintla Tacubaya 4, 33

Iriarte B., Johnson H.L., Mitchell R.I., et al., 1965, Sky \& Telescope 30, 21

Jaschek M., Egret D., 1982, Catalogue of Stellar Groups, SPCDS 4, Obs. Strasbourg

Jaschek M., Jaschek C., Hubert-Delplace A.M., Hubert H., 1980, A\&AS 42, 103

Jeong J.H. Lee Y.S., 1988, Vistas Astron. 31, 287

Johnson H.L., 1964, Bol. Inst. Tonantzintla Tacubaya 3, 305 
Johnson H.L., Harris III D.L., 1954, ApJ 120, 196

Johnson H.L., 1958, Lowell Obs. Bull. 4, 37

Johnson H.L., 1965, Comm. Lunar Plan. Lab. 3, 73

Johnson H.L., Mitchell R.I., 1975, Rev. Mex. Astron. Astrofis. 1,299

Johnson H.L., Mitchell R.I., Iriarte B., 1966, Comm. Lunar Plan. Lab. 4, 99

Johnson H.L., Mitchell R.I., Latham A.S., 1967, Comm. Lunar Plan. Lab. 6, 85

Johnson H.L., Morgan W.W., 1951, ApJ 114, 552

Johnson H.L., Morgan W.W., 1953, ApJ 117, 313

Juza K., Harmanec P., Božič H., et al., 1994, A\&AS 107, 403

Kaiser D., 1989, A\&A 222, 187

Kalv P., 1977, Inf. Bull. Var. Stars 1359

Kaper L., van Kerkwijk M., 1992, Be Star Newslett. 25, 11

Katahira J., Hirata R., Ito M., et al., 1996, PASJ 48, 317

Kiehling R., 1984 (priv. comm. in A\&A 138, 140)

Kilkenny D., Whittet D.C.B., Davies J.K., et al., 1985, South Afr. Astron. Obs. Circ. No. 9. 55

Kogure T., 1989, Ap\&SS 163, 7

Kogure T., Hirata R., 1982, Bull. Astr. Soc. India 10, 281

Kozok J.R., 1984 (priv. comm. in A\&A 138, 140)

Kozok J.R., 1985, A\&AS 61, 387

Koubsky P., Gulliver A.F., Harmanec P., et al., 1989, Bull. Astron. Inst. Czech. 40, 31

Kurucz R.L., Peytremann E., Avrett E.H., 1974, Blanketed Model Atmospheres for Early-Type Stars. Smithsonian Institution Press

Kurucz R.L., 1992, Model Atmospheres, magnetic tape

Lake R., 1965, Mon. Not. Astr. Soc. S. Africa 24, 41

Landolt A.U., 1969, PASP 81, 443

Landolt A.U., 1971, PASP 83, 650

Lanz T., 1986, A\&AS 65, 195

Lee T.A., 1968, ApJ 152, 913

Lenouvel F., Daguillon J., 1956, J. Obs. 39, 1

Lesh J.R., 1968, ApJS 17, 371

Lindroos K.P., 1983, A\&AS 51, 161

Ljunggren B., Oja T., 1964, Ark. Astron. 3, 439

Loden L.O., 1969, Ark. astr. 5, 149

Lucke P.B., 1974, ApJS 28, 73

Lutz J.H., Lutz T.E., 1972, AJ 77, 376

Lutz T.E., Lutz J.H., 1977, AJ 82, 431

Lynas-Gray A.E., Hill P.W., 1979, MNRAS 189, 777

Lynga G., 1959, Ark. Astr. 2, 379

McNamara B.J., 1976, PASP 88, 144

McNamara B.J., 1987, ApJ 312, 778

Malmquist K.G., Ljunggren B., Oja T., 1960, Ann. Uppsala Astr. Obs. 4, No. 8.

Manfroid J., Sterken C., Bruch A., et al., 1991, A\&AS 87, 481

Manfroid J., Sterken C., Cunow B., et al., 1995, A\&AS 109, 329

Margon B., Bowyer S., Panegor G., 1976, MNRAS 176, 217

Mathis J.S., 1990, ARA\&A 28, 37

Mendoza V.E.E., 1958, ApJ 128, 207

Mennickent R.E., Vogt N., 1991, Rev. Mex. Astron. Astrofis. 22,310

Mennickent R.E., Vogt N., Sterken C., 1994, A\&AS 108, 237

Menzies J.W., Marang F., Westerhuys J.E., 1990, South Afr. Astron. Obs. Circ. No. 14. 33

Mermilliod J.C., Mermilliod M., 1994, Catalogue of Mean $U B V$ Data on Stars. Springer-Verlag
Merrill P.W., Burwell C.G., 1949, ApJ 110, 387

Mitchell R.I., 1960, ApJ 132, 68

Moffet T.J., Barnes III T.G., 1979a, Inf. Bull. Var. Stars 1533

Moffet T.J., Barnes III T.G., 1979b, PASP 91, 180

Mon M., 1984, Thèse de troisième cycle, Paris VII, unpublished

Mook D.E., Boley F.I., Foltz C.B., et al., 1974, PASP 86, 894

Moreno H., 1971, A\&A 12, 442

Nakagiri M., Hirata R., 1979, Inf. Bull. Var. Stars 1565

Nordh H.L., Olofsson S.G., 1977, A\&A 56, 117

Norton A.J., Coe M.J., Estela A., et al., 1991, MNRAS 253, 589

Oblak E., Chareton M., 1980, A\&AS 41, 255

Oja T., 1991, A\&AS 89, 415

Osawa K., Hata S., 1960, Ann. Tokyo Astr. Obs. 2nd Ser. 6, 148

Osawa K., Hata S., 1962, Ann. Tokyo Astr. Obs. 2nd Ser. 7, 209

Papousek J., 1979, Scripta Fac. Sci. Nat. Univ. Purkynianae, Brunensis phys. 9,75

Pavlovski K., Harmanec P., Božič H., et al., 1996, A\&A (preprint)

Pavlovski K., Ružič Z., 1990, A\&A 236, 393

Penprase B.E., 1992, ApJS 83, 273

Penston M.J., 1973, MNRAS 164, 133

Percy J.R., 1986, PASP 98, 342

Percy J.R., Coffin B.L., Drukier G.A., et al., 1988, PASP 100, 1555

Perry C.L., Olsen E.H., Crawford D.L., 1987, PASP 99, 1184

Peton A., 1981, A\&A 101, 96

Peters G.J., 1989, Be Star Newslett. 21, 9

Peters G.J., 1990, Be Star Newslett. 22, 19

Peters G.J., 1991, Be Star Newslett. 23, 14

Peters G.J., 1992, Be Star Newslett. 25, 10

Peters G.J., 1994, Be Star Newslett. 27, 13

Pfleiderer J., Dachs J., Haug U., 1966, Z. Astrophys. 64, 116

Philip A.G.D., Philip K.D., 1973, AJ 179, 855

Poretti E., 1982, Inf. Bull. Var. Stars 2129

Quirrenbach A., Bjorkman K.S., Bjorkman J.E., et al., 1997, ApJ 479, 477

Reynolds A.P., Hilditch R.W., Bell S.A., et al., 1992, MNRAS 258, 439

Roche P., Coe M.J., Fabregat J., et al., 1993, A\&A 270, 122

Roman N.G., 1955, ApJS 2, 195

Rucinski S.M., 1987, PASP 99, 487

Rufener F., 1979, Geneva Photometric System: Catalogue of Standard Stars. In: Philip A.G.D (ed.), Problems of Calibration of Multicolor Photometric Systems, Dudley Observatory Reports No. 14., p. 443

Rufener F., 1980, Third Catalogue of Stars Measured in the Geneva Observatory Photometric System, Observatoire de Genève

Rufener F., 1981, A\&AS 45, 207

Rufener F., 1988, Catalogue of Stars Measured in the Geneva Observatory Photometric System (4th edition), Observatoire de Genève

Rufener F., Maeder A., 1971, A\&AS 4, 43

Rufener F., Nicolet B., 1988, A\&A 206, 357

Salukvadze G.N., Javakhishvili G.S., 1995, Astron. Nachr. 316, 275

Schild R.E., 1973, ApJ 179, 221

Schild R.E., 1978, ApJS 37, 77 
Schuster W.J., 1976, Rev. Mex. Astron. Astrofis. 1, 327

Schuster W.J., Alvarez M., 1983, PASP 95, 141

Schneider H., 1987, A\&AS 67, 545

Schuster W.J., Guichard J., 1984, Rev. Mex. Astron. Astrofis. 9,141

Searle L., 1958, ApJ 128, 61

Sharov A.S., Lyutyi V.M., 1988, AZh 65, 593

Sharov A.S., Lyutyi V.M., 1992, AZh 69, 544

Sharpless S., 1952, ApJ 116, 251

Shobbrook R.R., 1984, MNRAS 211, 659

Simonson S. Ch. III, 1968, ApJ 154, 923

Slawson R.W., Hill R.J., Landstreet J.D., 1992, ApJS 82, 117

Slettebak A., 1982, ApJS 50, 55

Slettebak A., Collins G.W. II, Boyce P.B., et al., 1975, ApJS 29,137

Sowell J.R., Wilson J.W., 1993, PASP 105, 36

Stagg C., 1987, MNRAS 227, 213

Stee Ph., Araujo F.X., Vakili F., et al., 1995, A\&A 300, 219

Stee Ph., Vakili F., Bonneau D., et al., 1997, A\&A (preprint)

Sterken C., Manfroid J., 1992, Astronomical Photometry - A Guide, Kluwer Acad. Publ.

Sterken C., Manfroid J., Anton K., et al., 1993, A\&AS 102, 79

Sterken C., Manfroid J., Beele D., et al., 1995, A\&AS 113, 31

Sterken C., Vogt N., Mennickent R., 1994, A\&A 291, 473

Stokes N.R., 1972, MNRAS 160, 155

Stoy R.H., 1963, Mon. Not. Astr. Soc. S. Africa 22, 157

Stoy R.H., 1968, Mon. Not. Astr. Soc. S. Africa 27, 119

Strömgren B., 1966, Ann. Rev. Astron. Astrophys. 4, 433

Štefl S., Baade D., Harmanec P., et al., 1995, A\&A 294, 135

Thé P.S., Wesselius P.R., Janssen I.M.H.H., 1986, A\&AS 66, 63

Thomas R.N., 1983, Stellar Atmospheric Structural Pattern
(NASA-CNRS), NASA SP-471

Tüg H., 1980, A\&AS 39, 67

Tempesti P., Patriarca R., 1976, Inf. Bull. Var. Stars 1164

Turner D.G., 1976, ApJ 210, 65

Turon C., Crézé M., Egret D., et al., 1992, The Hipparcos Input Catalogue, ESA SP-1136

van der Wal P.B., Nagel C., Voordes H.R., et al., 1972, A\&AS 6,131

Vogt N., 1976, A\&A 53, 9

Wackerling L.R., 1972, PASP 84, 827

Warman J., Echevarría J., 1977, Rev. Mex. Astron. Astrofis. 3,133

Warren W.H., Hesser J.E., 1978, ApJS 36, 497

Westerlund B.E., 1963, MNRAS 127, 71

Westin T.N.G., 1982, A\&AS 49, 561

Wiegandt R., 1984 (priv. comm. in A\&A 138, 140)

Zelwanowa E., Schoneich W., 1971, Astr. Nach. 293, 155

Zorec J., 1985, Rev. Mex. Astron. Astrofis. 10, 277

Zorec J., 1986, Thèse d'État, Université Paris VII

Zorec J., 1994, The Photosphere of Pleione. In: Balona L.A., Henrichs H.F., Le Contel J.M. (eds.), Pulsation, Rotation and Mass Loss in Early-Type Stars, IAU Symp. No. 162. Kluwer Acad. Publ., p. 362

Zorec J., Briot D., 1985, Rev. Mex. Astron. Astrof. 10, 317

Zorec J., Briot D., 1991, A\&A 245, 150

Zorec J., Briot D., Divan L., 1983, A\&A 126, 192

Zorec J., Garcia A., 1991, Correlation between the Paschen emission lines and $\mathrm{H} \alpha$ of Be stars. In: Jaschek C., Andrillat Y. (eds.), The Infrared Spectral Region of stars. Cambridge Univ. Press, p. 388

Zorec J., Höflich P., Divan L., 1989, A\&A 210, 279 